\title{
A piezoelectric based energy harvester with dynamic magnification: modelling, design and experimental assessment
}

Davide Castagnetti, $1 \square$

Email davide.castagnetti@unimore.it

Enrico Radi, 1

1 Department of Sciences and Methods for Engineering, University of Modena and Reggio Emilia, 42122 Reggio Emilia, RE, Italy

Received: 20 October 2017 / Accepted: 15 May 2018

\begin{abstract}
This work presents a simple and innovative piezoelectric energy harvester, inspired by fractal geometry and intrinsically including dynamic magnification. Energy harvesting from ambient vibrations exploiting piezoelectric materials is an efficient solution for the development of selfsustainable electronic nodes. After an initial design step, the present work investigates the eigenfrequencies of the proposed harvester, both through a simple free vibration analysis model and through a computational modal analysis. The experimental validation performed on a prototype, confirms the accurate frequency response predicted by these models with five eigenfrequencies below $100 \mathrm{~Hz}$. Despite the harvester has piezoelectric transducers only on a symmetric half of the top surface of the lamina, the rate of energy conversion is significant for all the investigated eigenfrequencies. Moreover, by adding a small ballast mass on the structure, it is possible to excite specific eigenfrequencies and thus improving the energy conversion.
\end{abstract}

AQ1

\section{Keywords}

Energy harvester

Dynamic magnification

Piezoelectric

Multi-frequency 
Experimental

Free vibration model

\section{Introduction}

Harvesting energy from ambient vibrations, exploiting the direct piezoelectric effect, is an efficient solution for powering self-sustainable electronic devices and remote sensors.

The integration of an energy harvester in these devices, eliminates the need for external power sources (i.e. batteries or connection to the power grid), thus ensuring continuous operation over a long period of time.

Among the large number of piezoelectric energy harvesters proposed in the literature, a peculiar approach consists in equipping the harvester with a dynamic magnifier. Dynamic magnification of the ambient energy that excites the harvester amplifies the dynamic response of the device (strain or displacement), thus increasing the harvested energy. Basically, dynamic magnification consists in constraining the harvester to an intermediate spring-mass system, which is fixed to the vibrating base structure.

Aldraihem and Baz [1] provide a rigorous analysis of the dynamic magnifier concept applied to a single degree of freedom harvester, but solutions incorporating this concept can be found in previous works. Cornwell et al. [2], Rastegar et al. [3] and Ma et al. [4] propose harvesters which incorporate an intermediate elastic system between the oscillating base and a cantilevered piezoelectric structure. The proper tuning of the whole elastic system makes possible a substantial improvement of the output power, thank to dynamic magnification of the input oscillation. Lee et al. [5] investigate a segment type harvester which utilizes multiple modes by separating the piezoelectric material. Yang et al. [6] present a wideband piezoelectric harvester consisting of two elastically and electrically connected cantilevers. Erturk et al. [7] propose a new L-shaped beam-mass structure piezoelectric energy harvester exhibiting two very close natural frequencies. Xu et al. [8] investigate a similar configuration consisting of a cantilever with an extended orthogonal auxiliary part inducing a uniform strain distribution in the piezoelectric element. Tang et al. [9] analytically investigate a dual-mass vibration energy harvester that exhibits a higher conversion efficiency than a traditional single degree-of freedom solution simply by connecting two masses in series. Following the work by Aldraihem and Baz [1], Aladwani et al. [10], [11] demonstrate analytically and numerically that the class of cantilevered piezoelectric energy harvester with dynamic magnifier is a simple, feasible and effective mean for improving the performance 
of a traditional cantilevered converter. Nou et al. [12] increase the electric energy harnessed from a thermoacoustic-piezoelectric harvester through a configuration including a dynamic magnifier. Seo et al. [13] present a piezoelectric energy harvester deploying a flexible body beam in order to reduce the stiffness of the device, thus shifting down the high mode resonant frequencies into a usable range. Zhou et al. [14] propose a highly efficient piezoelectric energy harvester composed by a multi-mode intermediate beam with a tip mass (acting as a dynamic magnifier), and by an energy harvesting beam. Vasic and Costa [15] investigate a similar configuration, of a piezoelectric energy harvester consisting of an intermediate beam with a tip mass acting as dynamic magnifier. Dhakar et al. [16] present an energy harvester comprising a piezoelectric bimorph coupled at the end with a polymer beam including a tip mass. Kim et al. [17] examine the power enhancement obtained with a piezoelectric vibration energy harvester consisting of an auxiliary frequencytuned mass unit and an harvesting unit. Sun et al. [18] experimentally assess one of the configuration proposed in [17], consisting in a small-form-factor piezoelectric vibration based energy harvester. Sharma et al. [19] study the influence of the cross-section of the dynamic magnifier on energy harvesting. O'Donoghue et al. [20] and Nico et al. [21], [22] propose and investigate an innovative multi-degree of freedom velocity amplified harvester.

Three are the main drawbacks of the configurations proposed in the literature: first, a complex geometry; second, large dimensions and a non-compact (long cantilevers) shape; third, energy conversion for a small number of eigenfrequencies distributed in a large range and within a narrow bandwidth.

In order to overcome these drawbacks, the present work presents, analyzes, and experimentally investigates an innovative vibration-based piezoelectric energy harvester intrinsically including dynamic magnification and featuring a fractalinspired geometry.

Four are the steps of the work. The first step presents the innovative structure for the energy harvester, which was initially proposed in [23], [24] among a set of fractal-inspired multi-frequency structures [25, 26, 27]. Specifically, Ref. [23] presents four different fractal-inspired structures for energy harvesting, the papers [25] - [27] extensively investigates two of these structures, while the work in [24] presents a preliminary study of the energy harvester with dynamic magnification. Compared to Ref. [24], The original contribution of the present paper consists in the detailed analytical and computational modelling of the structure combined with a deeper experimental investigation. The second step of the present work deals with analytical and computational modelling of the eigenfrequencies and eigenmodes of the harvester prototype, while the third step 
focuses on the experimental assessment of the system. The work ends with the analysis and discussion of the results, showing the multi-frequency response of the proposed harvester, together with a high conversion efficiency.

\section{Conceptual solution for the energy harvester}

Figure 1 shows the innovative and simple conceptual solution for the piezoelectric energy harvester presented in this paper. This structure features a fractal-inspired geometry obtained by a square cantilever plate cut in order to create a peculiar zigzag path between the two built-in constraints, described in grey.

\section{Fig. 1}

Structure of the proposed fractal-inspired geometry featuring dynamic magnification

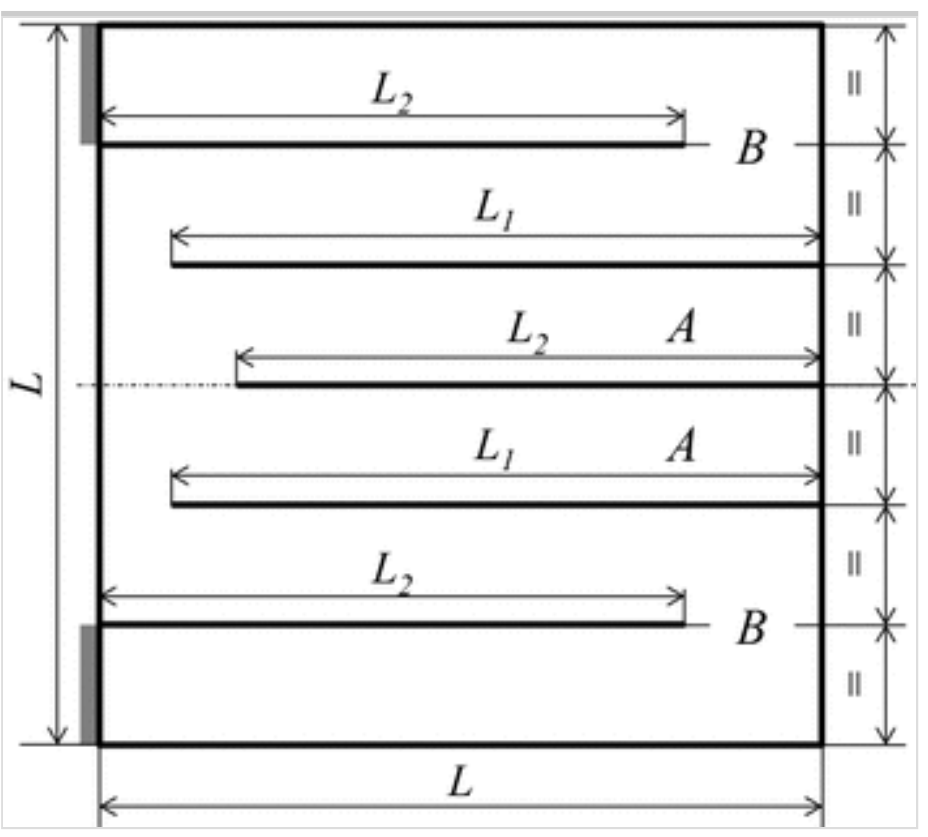

The length, $L_{i}$, of each cut was calculated according to the following simple relationship:

$$
L_{i}=0.9^{i} L
$$

where the exponent $i$ is an integer number, $L$ is the side length of the square lamina, and the 0.9 coefficient allows to obtain a robust structure and gives the possibility to perform many iteration levels in the fractal geometry. Figure 1 describes a fractal-inspired structure obtained for $i=1$ and 2, while Fig. 2 displays a similar structure, featuring an additional fractal iteration level $(i=1,2$ and 3). By focusing on Fig. 1, two $S$-shaped structures $(B)$ connect the fixed ends of the inner cantilevers $(A)$ to the external constraints. It follows that these $S$ - 
shaped structures $(B)$ act as a spring-mass system between the cantilevers $(A)$ and the external vibrating base, thus allowing the dynamic magnification of the strain experienced by the inner cantilevers, as described in [10]. Similarly, thanks to its complex structure, the solution in Fig. 2 features multiple levels of dynamic magnification.

Fig. 2

Second iteration level of the fractal geometry proposed in Fig. 1

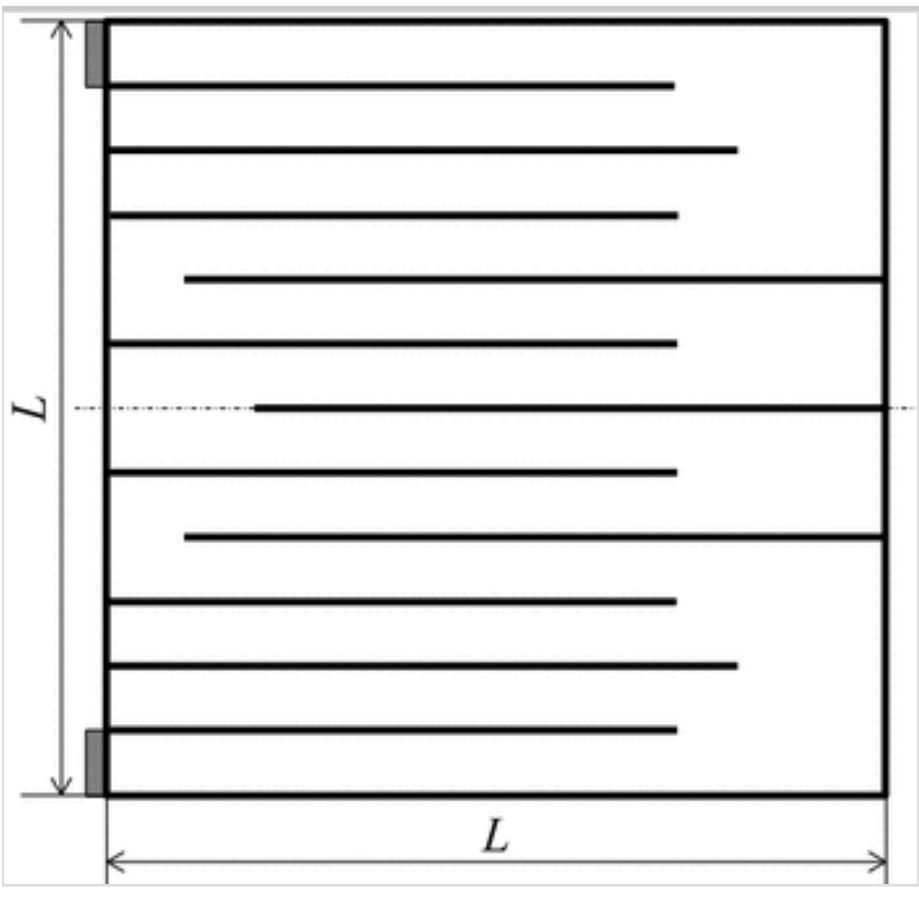

\section{Modelling and prototype of the harvester}

This section involves four steps, from a simple analytical free vibration model of the proposed structure up to the detailed design of the harvester prototype. The first step proposes a simple free vibrations analysis model of the structure, to orient the design of the system. The second step develops a FE model for a peculiar configuration of the structure, aimed at predicting its eigenfrequencies and the corresponding eigenmodes. This step allows us to identify where the maximum strains occur, and to define, in the third step, the exact prototype configuration. The fourth step investigates the modal response and the dynamic analysis of the energy harvester prototype, through a detailed FE simulation. All the FE analyses were performed with the ABAQUS 6.12 software [28].

\subsection{Free vibration model}

Figure 3 describes the conceptual sketch of the structure in Fig. 1, used to develop the free vibration model. Since the thickness to length ratio of an energy 
harvester is typically equal to $1 / 100$, the classical Euler-Bernoulli beam theory is used. With exception of the short connections $A, B, C, D$, and $E$ that, with reasonable approximation, were assumed as rigid, the model describes bending and twisting of all the beams.

Fig. 3

Sketch of the strucure for the analytical free vibration model

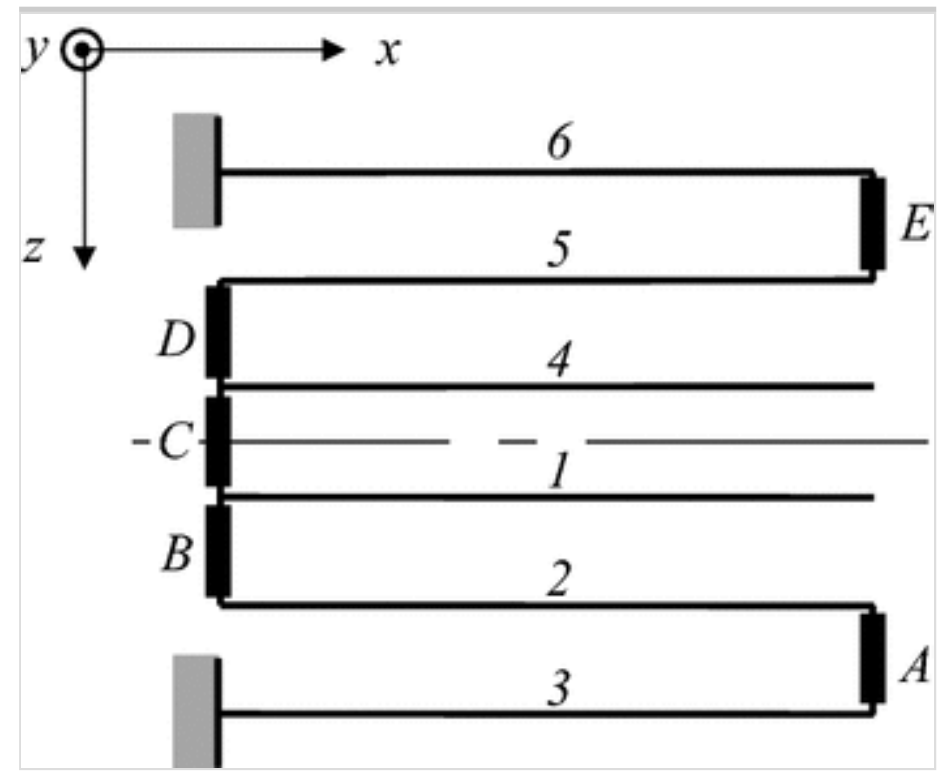

For the $i$-th beam $(i=1,2, \ldots, 6)$ we can define two functions, which depend on the axial coordinate of the beam (along the $x$ direction in Fig. 3), and on time, $t$. Namely, the function $v_{i}(x, t)$ that gives the instantaneous transverse displacement (in the $y$ direction in Fig. 3) of the center of elasticity of the cross section of the $i$-th beam, and the function $\varphi_{i}(x, t)$ that gives the instantaneous bending rotation of the cross section of the $i$-th beam. According to [29], [30], the equations of motion for the free vibrations of an Euler-Bernoulli beam in the transverse direction and that describing torsional vibrations are:

$$
\begin{gathered}
\rho A \frac{\partial^{2} v_{i}(x, t)}{\partial t^{2}}+E I \frac{\partial^{4} v_{i}(x, t)}{\partial x^{4}}=0 \\
J_{0} \rho \frac{\partial^{2} \varphi_{i}(x, t)}{\partial t^{2}}-G J_{t} \frac{\partial^{2} \varphi_{i}(x, t)}{\partial x^{2}}=0
\end{gathered}
$$

respectively, where $\rho$ is the material density, $A$ is the area of the cross section of the beam, $E$ the Young's modulus of the beam material, $I$ is the inertia moment about the $z$ axis (Fig. 3) of the beam cross-section, $J_{0}$ is the axial moment of inertia of the beam cross-section (about the $x$ axis in Fig. 3), $J_{t}$ is the torsional 
constant of the beam cross section, $E$ and $G$ are the Young and shear elastic moduli.

According to standard separation of variables technique, we can look at the solution of Eqs. (2) and (3) as the product of two functions, namely, a function of the position $x$ along the beam and a harmonic function of time $t$ :

$$
\begin{aligned}
& v_{i}(x, t)=V_{i}(x) \sin \left(\omega_{n} t\right) \\
& \varphi_{i}(x, t)=\theta_{i}(x) \sin \left(\omega_{n} t\right)
\end{aligned}
$$

where $V_{i}(x)$ and $\theta_{i}(x)$ are the amplitudes of the transverse displacement and angular rotation, respectively, of the $i$-th beam, while $\omega_{n}$ is the circular frequency of the $n$-th eigenmode of the structure.

The substitution of Eqs. (4) and (5) into Eqs. (2) and (3), respectively, yields the following linear ordinary differential equations:

$$
\begin{gathered}
V_{i}^{I V}(x)-\beta_{n}^{4} V_{i}(x)=0 \\
\theta_{i}^{I I}(x)+\alpha_{n}^{2} \theta_{i}(x)=0
\end{gathered}
$$

where the Roman superscript indicates the differentiation order with respect to the abscissa $x$, while the terms $\beta_{n}$ and $\alpha_{n}$ are defined, respectively, as:

$$
\begin{gathered}
\beta_{n}^{4}=\frac{\rho A}{E I} \omega_{n}^{2} \\
\alpha_{n}^{2}=\frac{J_{0}}{G J_{t}} \omega_{n}^{2}
\end{gathered}
$$

The solutions of the linear ordinary differential Eqs. (6) and (7) are:

$$
\begin{array}{cl}
V_{i}(x)=C_{i, 1 n} \cos \left(\beta_{n} x\right)+C_{i, 2 n} \sin \left(\beta_{n} x\right)+C_{i, 3 n} \cosh \left(\beta_{n} x\right)+C_{i, 4 n} \sinh \left(\beta_{n} x\right) & 10 \\
\theta_{i}(x)=D_{i, 1 n} \sin \left(\alpha_{n} x\right)+D_{i, 2 n} \cos \left(\alpha_{n} x\right) & 11
\end{array}
$$

respectively. Thus, there are six unknown coefficients $\left(C_{i, j n}, D_{i, k n}\right)$ for each beam: this requires eighteen boundary conditions to solve the model and identify the free vibrations of the structure. 


\subsection{Boundary, equilibrium and continuity conditions}

Two types of eigenmodes can occur on the structure: symmetric and antisymmetric eigenmodes. Therefore, splitting the solution into symmetric and antisymmetric modes allows us to study only one half of the structure. By assuming a rigid behavior for the connections $A, \ldots, E$, the following boundary, equilibrium and continuity conditions apply both to the symmetric and antisymmetric eigenmodes:

$V_{1}^{\prime \prime}(l)=0 \quad$ Vanishing of the tip bending moment of beam 1

$$
V_{1}^{\prime \prime \prime}(l)=0 \quad \text { Vanishing of the tip shear force of beam } 1
$$

$V_{2}^{\prime}(l)=V_{3}^{\prime}(l) \quad$ Same tip bending rotation between beam 2 and 3

$V_{2}^{\prime \prime}(l)=-V_{3}^{\prime \prime}(l) \quad$ Continuity of the tip bending moment between beam 2 and 315

$V_{2}^{\prime \prime \prime}(l)=-V_{3}^{\prime \prime \prime}(l) \quad$ Equilibrium of the tip shear force between beam 2 and 316

$V_{3}(0)=0 \quad$ Zero transverse displacement at the outer constrain of beam 3

$$
V_{3}^{\prime}(0)=0 \quad \text { Zero bending rotation at the outer constrain of beam } 3
$$

$V_{3}(l)=V_{2}(l)+\theta_{2}(l) b \quad$ Same transverse displacement at the tip of beam 2 and 3

$$
\theta_{1}^{\prime}(l)=0 \quad \text { Zero torque at the tip of beam } 1
$$

$$
\theta_{2}(l)=\theta_{3}(l) \quad \text { Same torsional rotation at the tip of beam } 2 \text { and } 3
$$

$$
\theta_{3}(0)=0 \quad \text { Zero torsional rotation at the outer constraint of beam } 3
$$

$G J_{t}\left(\theta_{2}^{\prime}(l)+\theta_{3}^{\prime}(l)\right)+E I V_{2}^{\prime \prime \prime}(l) b=0 \quad$ Equilibrium condition between bending $\mathrm{m}$ where $b$ is the width of the beam, while the equations concerning the boundary conditions at the midpoint of the structure are peculiar for the symmetric $V_{1}(0)=V_{2}(0) \quad$ Same transverse displacement at the root of beam 1 and 224 
$V_{1}^{\prime}(0)=V_{2}^{\prime}(0) \quad$ Same bending rotation at the root of beam 1 and 2

$V_{1}^{\prime \prime}(0)=-V_{2}^{\prime \prime}(0) \quad$ Continuity of the bending moment at the root of beam 1 and 2

$V_{1}^{\prime \prime \prime}(0)=-V_{2}^{\prime \prime \prime}(0) \quad$ Equilibrium of the shear force at the root of beam 1 and 227

$\theta_{1}(0)=0 \quad$ Zero torsional rotation at the root of beam 1

$\theta_{2}(0)=0 \quad$ Zero torsional rotation at the root of beam 2

or antisymmetric eigenmodes:

$$
\begin{gathered}
V_{1}(0)=\theta_{1}(0) b / 2 \quad \text { Transverse displacement at the root of beam } 1 \\
V_{2}(0)=\theta_{1}(0) 3 b / 2 \quad \text { Transverse displacement at the root of beam } 2 \\
V_{1}^{\prime}(0)=0 \quad \text { Bending rotation at the root of beam } 1 \\
V_{2}^{\prime}(0)=0 \quad \text { Bending rotation at the root of beam } 2
\end{gathered}
$$

$\theta_{1}(0)=\theta_{2}(0) \quad$ Continuity of torsional rotation at the root of beam 1 and 234

$2 G J_{t}\left(\theta_{1}^{\prime}(0)+\theta_{2}^{\prime}(0)\right)=E I\left(V_{1}^{\prime \prime \prime}(0)+3 V_{2}^{\prime \prime \prime}(0)\right) b \quad$ Equilibrium condition betwee

Overall, this set of equations defines the displacements, rotations, shear forces, bending moments and torque at the boundaries of the structure or at the beam connections.

\subsection{General solution}

Using Eqs. (12-35), we obtain two distinct linear algebraic systems for symmetric and antisymmetric eigenmodes, respectively. Each system involves eighteen unknowns $C_{i, j n}, D_{i, k n}$, according to the following matrix form:

$$
\mathbf{H} \times\left\{C_{1,1 n}, \ldots, C_{3,4 n}, D_{1,1 n}, \ldots, D_{3,2 n}\right\}^{T}=0
$$

where the square matrix $\mathbf{H}$ collects the coefficients of the set of equations. 
The linear system (36) has a non-trivial solution if and only if the determinant of the $\boldsymbol{H}$ matrix is null, thus giving the following characteristic transcendental equation of the system:

$$
\operatorname{det}(\mathbf{H})=0
$$

The infinite roots of Eq. (37) correspond to the infinite circular frequencies $\omega_{n}$ of the structure. For each circular frequency $\omega_{n}$ of the $n$-th eigenmode, using the set of linearly dependent Eqs. (36), it is possible to determine the $C_{i, j n}$, and $D_{i, k n}$ constants, by setting an arbitrary value for one of the unknown constants. Upon substitution of the parameters $C_{i, j n}$, and $D_{i, k n}$ in Eqs. (10) and (11), we finally obtain the expression of the eigenmodes associated to each circular frequency $\omega_{n}$.

\subsection{Assessment of the model}

In order to assess this free vibration analytical model, we compared it with a structural FE model developed through the ABAQUS 6.12 software. The model describes the whole structure as in Fig. 3, assuming a side length of $100 \mathrm{~mm}$ and a thickness of $0.8 \mathrm{~mm}$. The short beam connections $A$ through $E$ were described as rigid. Elsewhere, the model used three dimensional, linear beam elements (B33), and adopted a Young's modulus of $203000 \mathrm{MPa}$ and a Poisson's ratio of 0.3 for the steel material. Each part of the structure implemented the specific rectangular section, and built-in constraints were applied on the fixed ends. The analysis investigated the eigenfrequencies of the structure up to $1000 \mathrm{~Hz}$, using the Lanczos algorithm. Figure 4 shows the comparison between the eigenmodes and eigenfrequencies predicted by the analytical free vibration model and those from the corresponding FE model. The close agreement between the analytical and the FE model testifies the accuracy of the proposed model and confirms its applicability to predict the modal response of this structure for arbitrary values of the geometric parameters.

\section{Fig. 4}

Comparison between the eigenmodes and eigenfrequencies predicted by the analytical free vibration model, on the left, and those provided by the FE model, on the right for a side length of $100 \mathrm{~mm}$ and a transverse rectangular section $15 \mathrm{~mm}$ wide by $0.8 \mathrm{~mm}$ thick 

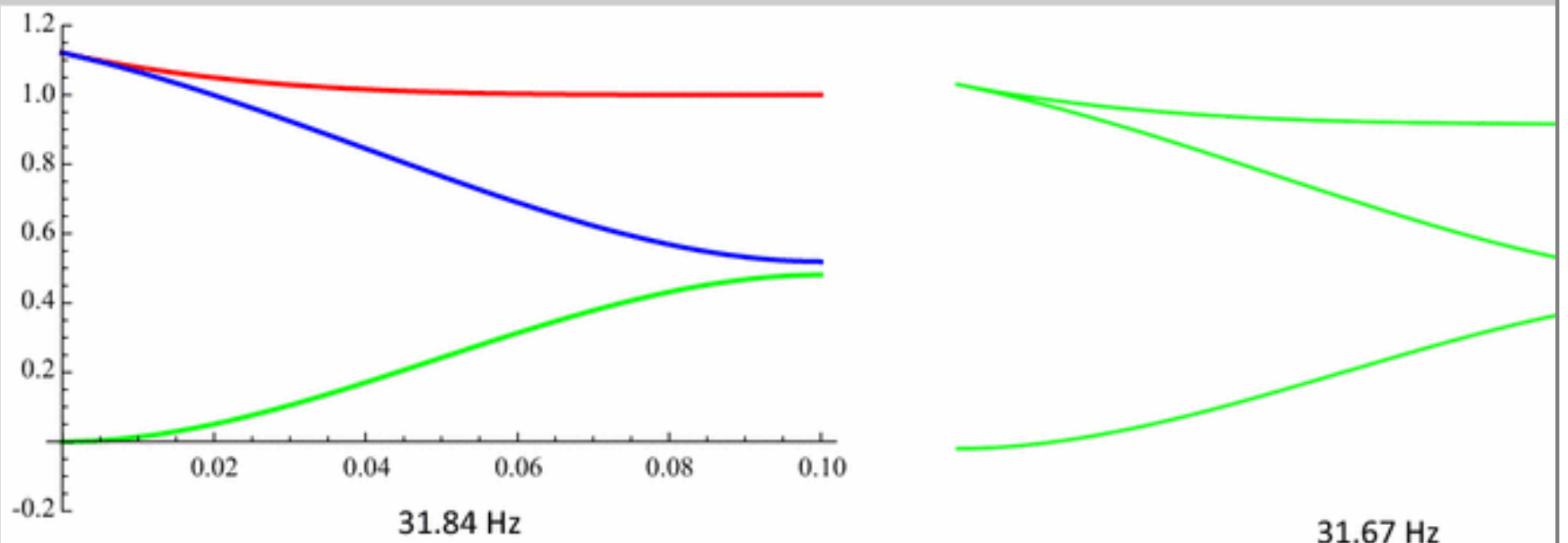

$31.67 \mathrm{~Hz}$

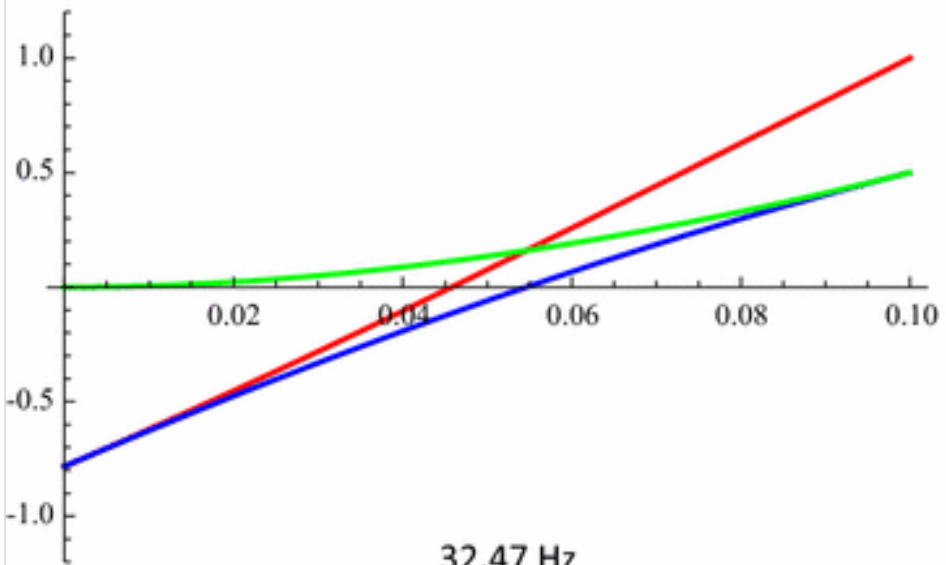

$32.47 \mathrm{~Hz}$

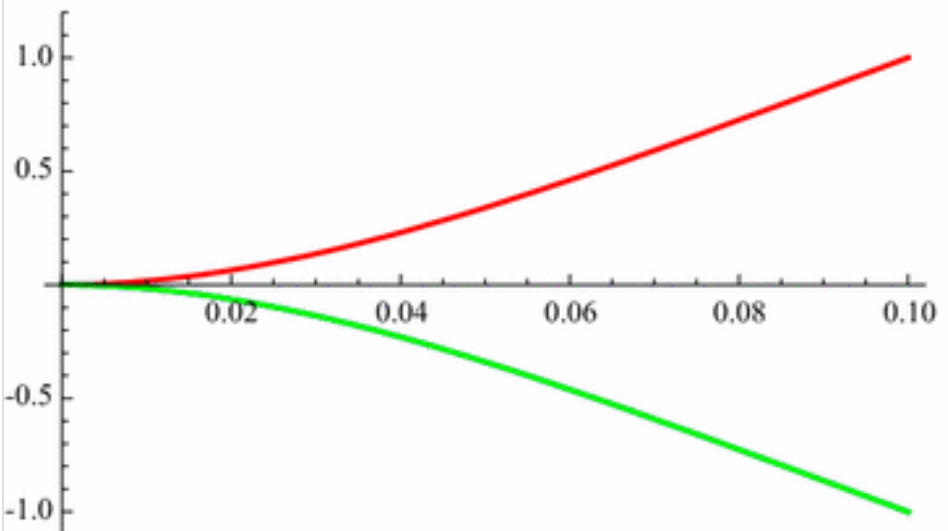

$65.68 \mathrm{~Hz}$

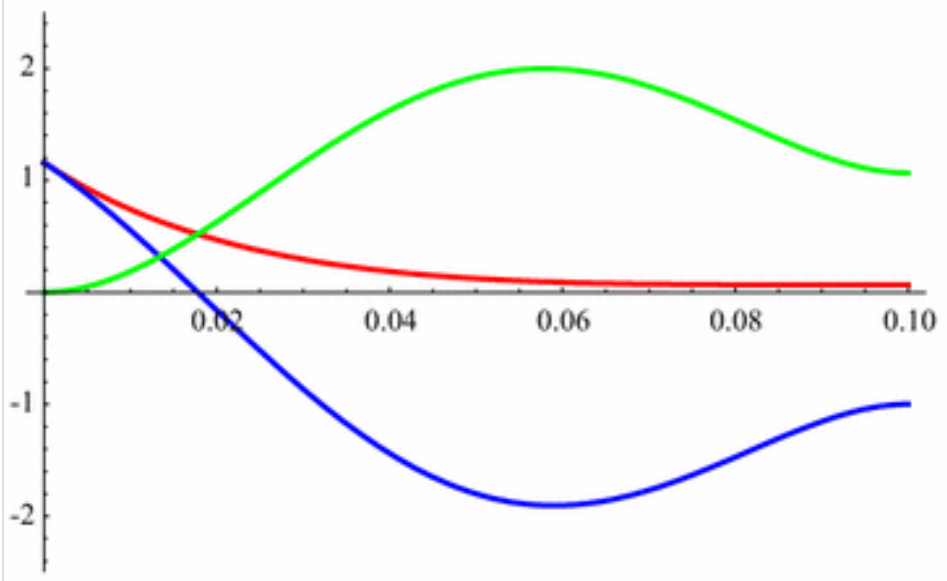

$340.81 \mathrm{~Hz}$

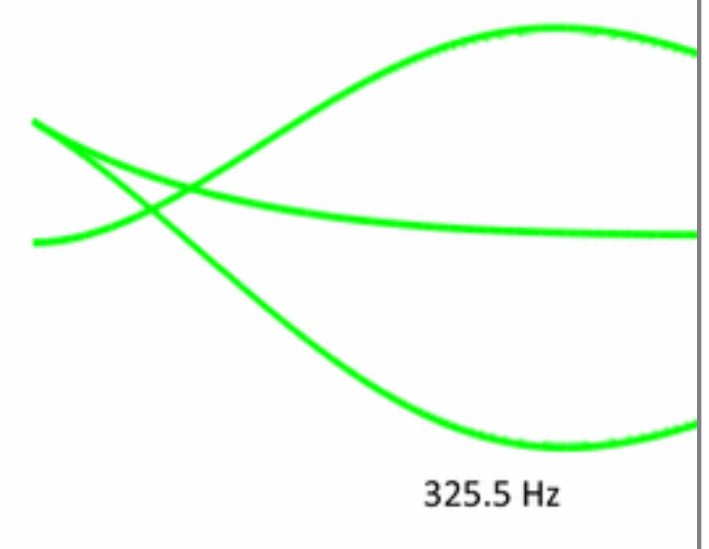




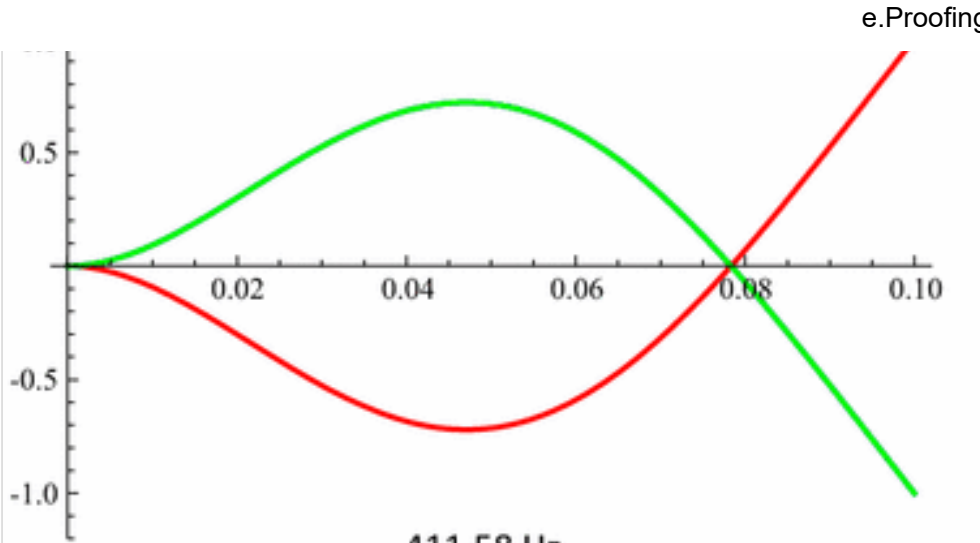

$411.58 \mathrm{~Hz}$

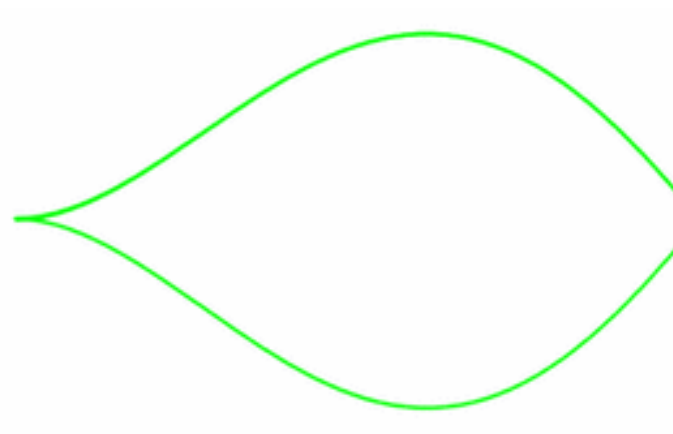

$411.25 \mathrm{~Hz}$

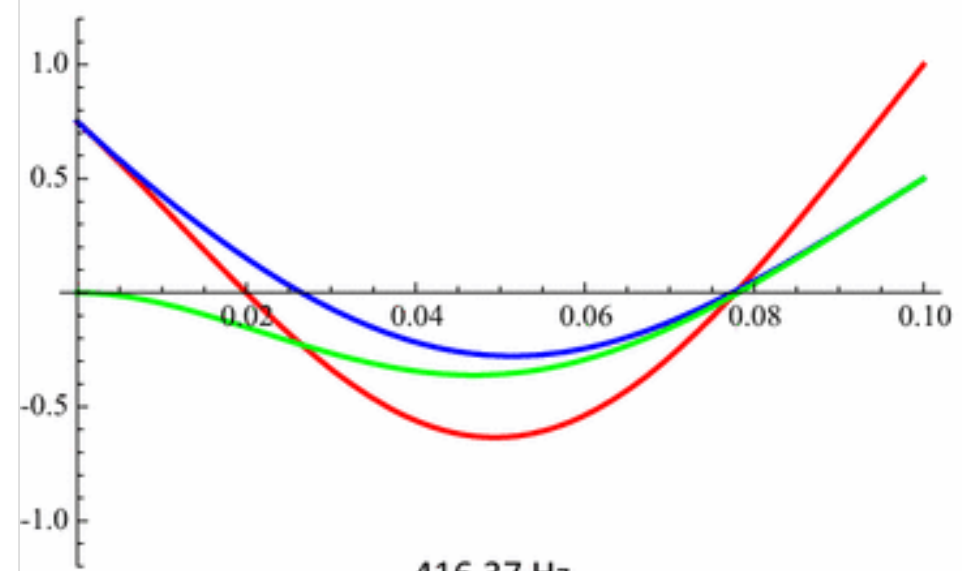

$416.37 \mathrm{~Hz}$

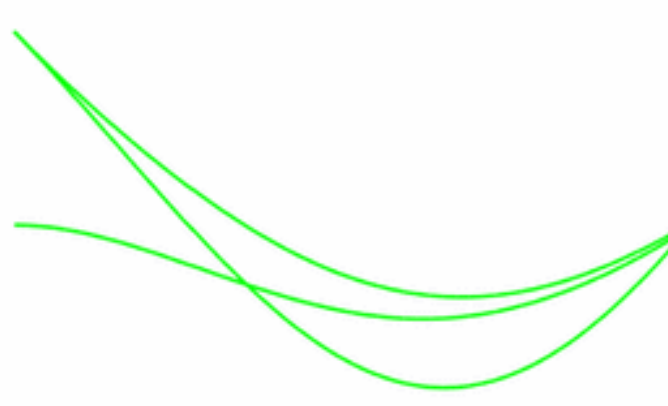

416.01

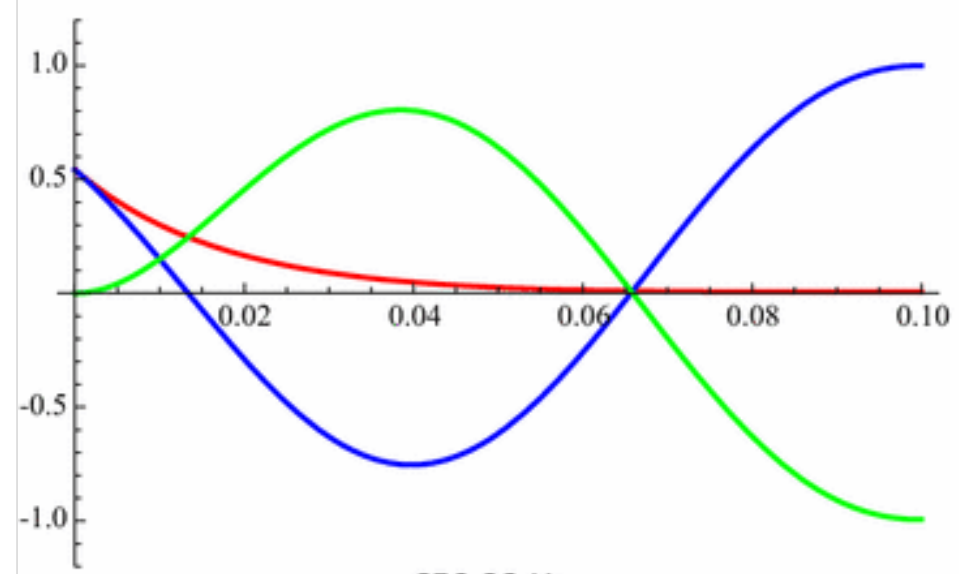

$650.22 \mathrm{~Hz}$

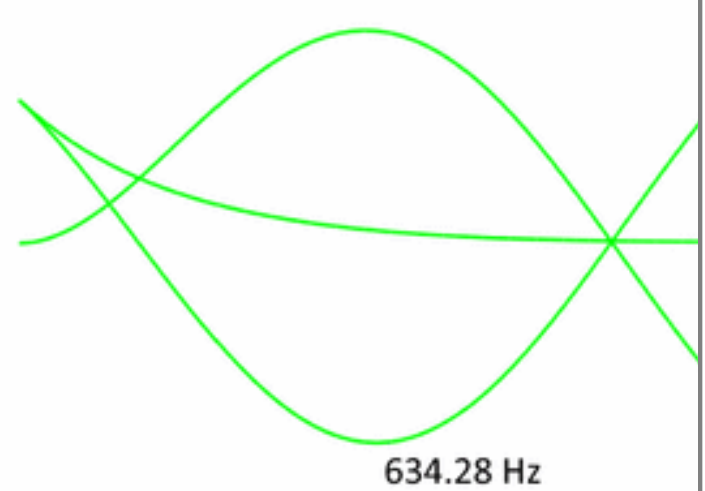

\subsection{Modal response prediction}

Table 1 shows how the side length $L$ and the thickness $s$ of the support structure affect the eigenfrequencies of the system, according to the predictions from the free vibration model. The table focuses on the eigenfrequencies below $1000 \mathrm{~Hz}$. It emerges that a structure with a side length $L$ equal to $100 \mathrm{~mm}$ and a thickness $s$ equal to $0.8 \mathrm{~mm}$ provides a very efficient device due to the large number of eigenfrequencies below $100 \mathrm{~Hz}$, which is the typical range of application for energy harvesting from ambient vibrations, and the small size of the structure. 


\section{Table 1}

Comparison between the eigenfrequencies for different values of side length, $L$, width, $b$, anı

\begin{tabular}{|c|c|c|c|c|c|c|c|c|}
\hline$L(\mathrm{~mm})$ & \multicolumn{3}{|l|}{50} & \multicolumn{3}{|l|}{100} & \multicolumn{2}{|l|}{150} \\
\hline$b(\mathrm{~mm})$ & \multicolumn{3}{|l|}{7} & \multicolumn{3}{|l|}{15} & \multicolumn{2}{|l|}{23} \\
\hline$s(\mathrm{~mm})$ & 0.04 & 0.08 & 1.20 & 0.04 & 0.08 & 1.20 & 0.04 & $0.0 \&$ \\
\hline \multirow{27}{*}{$\begin{array}{l}\text { Eigenfrequencies } \\
(\mathrm{Hz})\end{array}$} & 63.83 & 127.66 & 191.49 & 15.92 & 31.84 & 47.75 & 7.01 & 14.1 \\
\hline & 64.94 & 129.88 & 194.82 & 16.23 & 32.47 & 48.70 & 7.21 & 14. \\
\hline & 119.81 & 239.62 & 359.42 & 29.69 & 59.38 & 89.06 & 13.16 & 26. \\
\hline & 131.35 & 262.70 & 394.06 & 32.84 & 65.68 & 98.51 & 14.59 & 29.1 \\
\hline & 308.70 & 617.06 & 974.74 & 74.73 & 149.44 & 224.11 & 32.89 & 65. \\
\hline & 700.60 & & & 170.42 & 340.81 & 511.16 & 75.03 & 150 \\
\hline & 711.45 & & & 173.00 & 345.95 & 518.86 & 76.15 & 152 \\
\hline & 823.17 & & & 205.79 & 411.58 & 617.38 & 91.46 & 182 \\
\hline & 832.74 & & & 208.18 & 416.37 & 624.55 & 92.53 & 185 \\
\hline & 945.31 & & & 234.80 & 469.54 & 704.19 & 104.14 & 208 \\
\hline & & & & 325.20 & 650.22 & 974.85 & 143.06 & 286 \\
\hline & & & & 363.96 & 727.59 & & 159.89 & 319 \\
\hline & & & & 452.55 & 904.43 & & 197.61 & 395 \\
\hline & & & & 576.05 & & & 256.02 & 512 \\
\hline & & & & 576.22 & & & 256.10 & 544 \\
\hline & & & & 613.20 & & & 272.01 & 566 \\
\hline & & & & 639.40 & & & 283.20 & 616 \\
\hline & & & & 696.81 & & & 308.35 & 738 \\
\hline & & & & 849.10 & & & 369.49 & 842 \\
\hline & & & & 939.27 & & & 421.60 & 876 \\
\hline & & & & & & & 438.45 & \\
\hline & & & & & & & 501.85 & \\
\hline & & & & & & & 528.69 & \\
\hline & & & & & & & 554.21 & \\
\hline & & & & & & & 569.40 & \\
\hline & & & & & & & 605.30 & \\
\hline & & & & & & & 732.14 & \\
\hline
\end{tabular}




\subsection{Detailed modal analysis of the proposed structure}

An additional FE model (Fig. 5) describes in detail the configuration in Fig. 1 by means of four noded, doubly curved, thin shell elements (S4R5), with reduced integration and hourglass control [28]. The model assumes a side length $L$ equal to $100 \mathrm{~mm}$, a width of each inner beam of $15 \mathrm{~mm}$, and a thickness of $0.8 \mathrm{~mm}$ for the mild steel support square lamina. According to a preliminary convergence analysis, the average side length of the elements was set equal to $1 \mathrm{~mm}$. A Young's modulus of $206 \mathrm{GPa}$ and a Poisson's ratio of 0.3 describes the linear elastic behaviour of the material. As shown in Fig. 5, a built-in boundary condition along the edges reproduces the constraints in Fig. 1. The modal analysis investigates the range between 0 and $100 \mathrm{~Hz}$, using the Lanczos algorithm. For this frequency range, Fig. 6 shows the first five eigenmodes and the corresponding eigenfrequencies predicted by the FE model. In particular, the eigenmodes depict the contour map of the longitudinal strain.

\section{Fig. 5}

Finite element model of the proposed structure

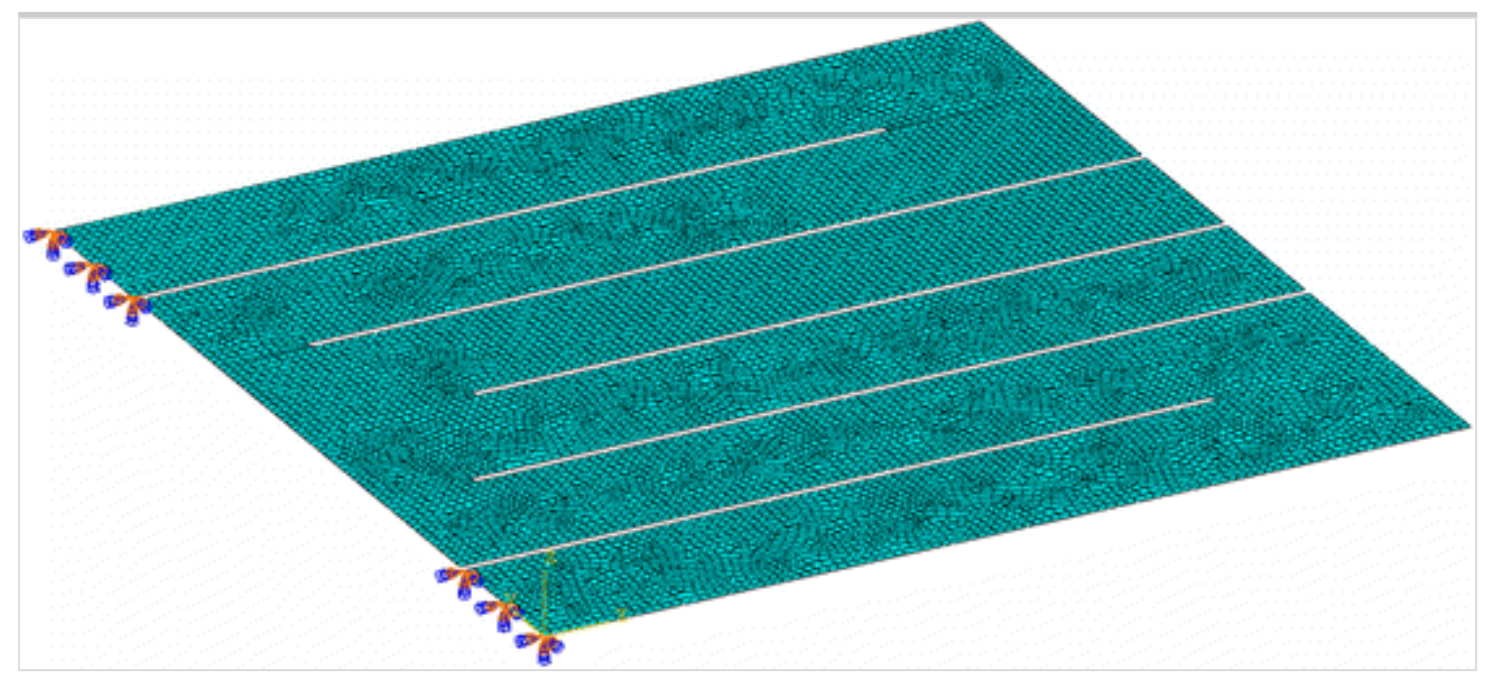


Fig. 6

FE prediction of the eigenmodes and corresponding eigenfrequencies of the proposed fractal-inspired geometry (Fig. 5) in the range below $100 \mathrm{~Hz}$

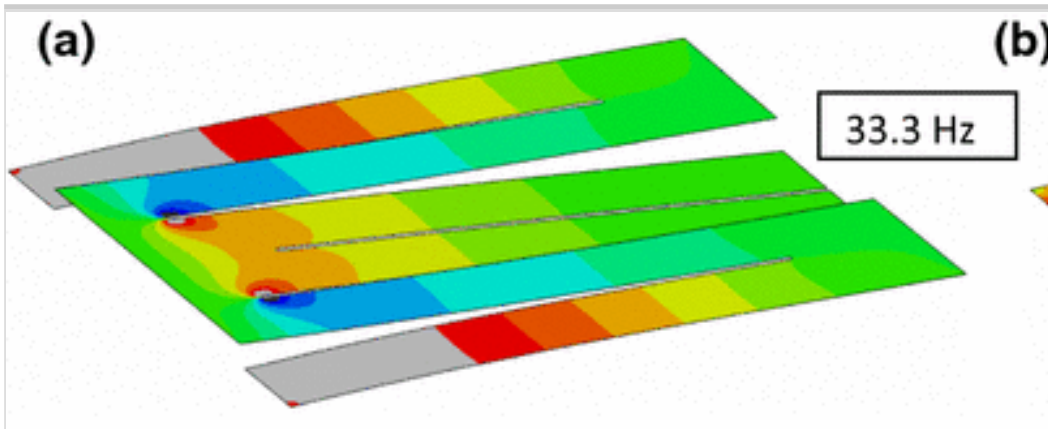

(b)

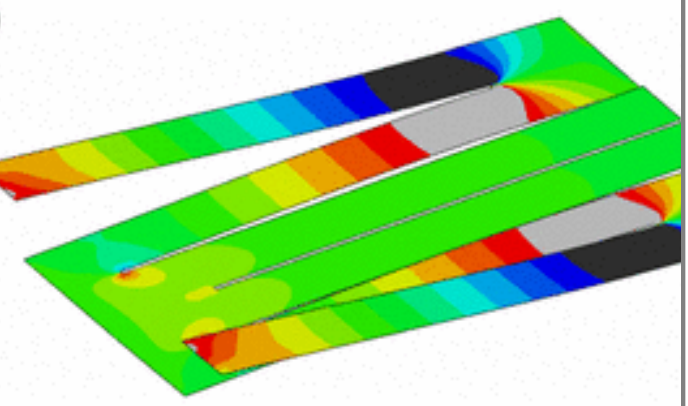

(c)

(d)
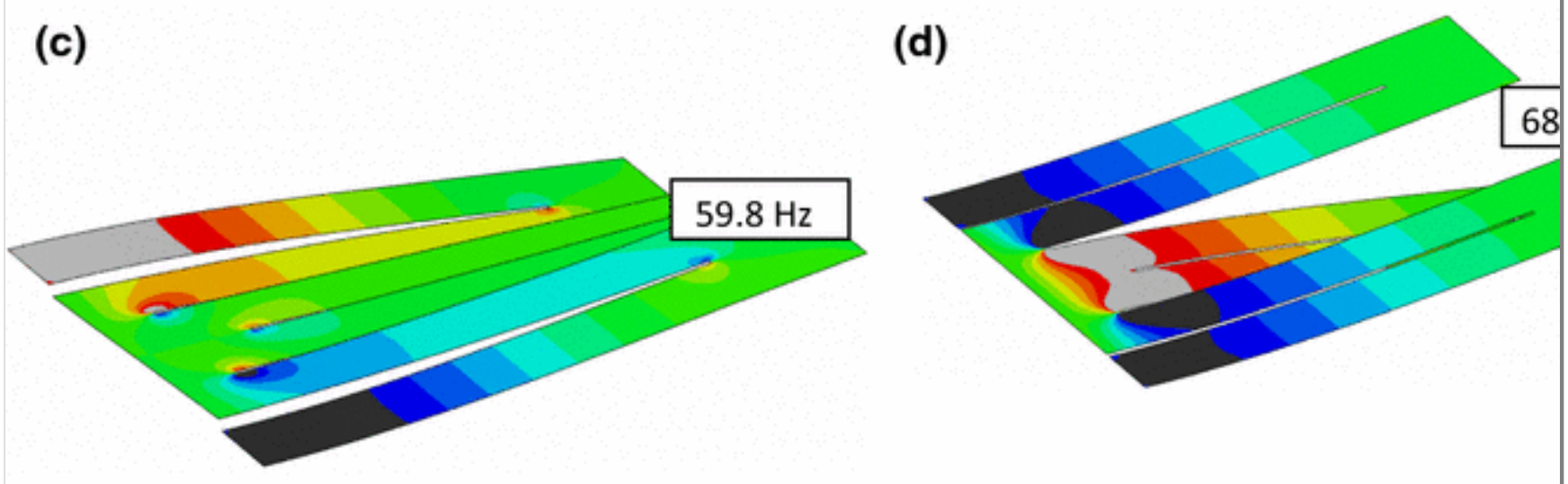

(e)

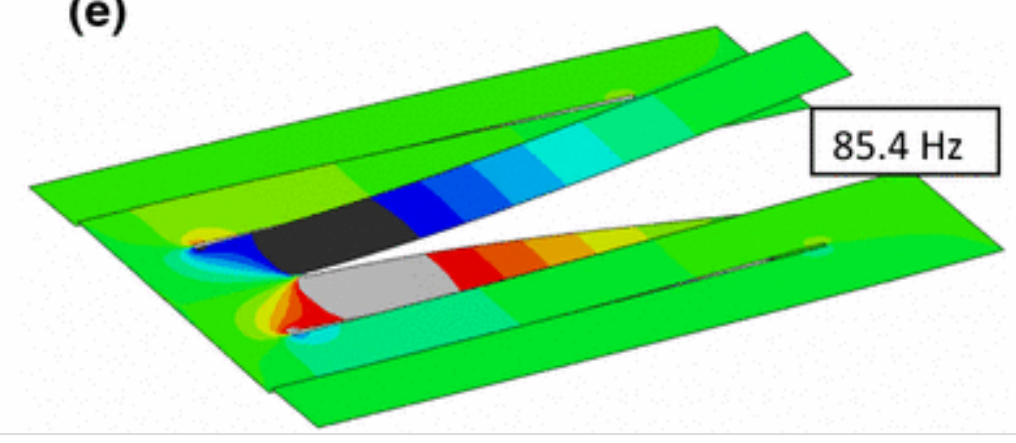

\subsection{Prototype design}

Figure 6 highlights some differences in the eigenfrequencies predicted by the detailed FE model in Fig. 5 compared to those provided by the analytical model for the same configuration (Fig. 4). This difference is imputable to the slightly different geometry between the two configurations: in Fig. 3 all the beams are $100 \mathrm{~mm}$ long, while the geometry in Fig. 5 is a square with a side length equal to $100 \mathrm{~mm}$. This peculiar geometry was designed in order to simplify the manufacturing of the prototype. By examining the five eigenmodes in Fig. 6, two main observations can be made. First, the deformation of the structure 
obviously occurs both in the dynamic magnifier element ( $B$ in Fig. 1$)$ and in the inner cantilevers ( $A$ in Fig. 1). Thus, both of them can be exploited to the aim of energy conversion. Second, the maximum bending strains occur within the three hatched zones in Fig. 7, where the piezoelectric transducers can be applied in order to maximize the energy conversion (from mechanical bending strain to electrical energy).

Fig. 7

Sketch of the prototype energy harvester, highlighting the position of the piezoelectric patches (hatched squares)

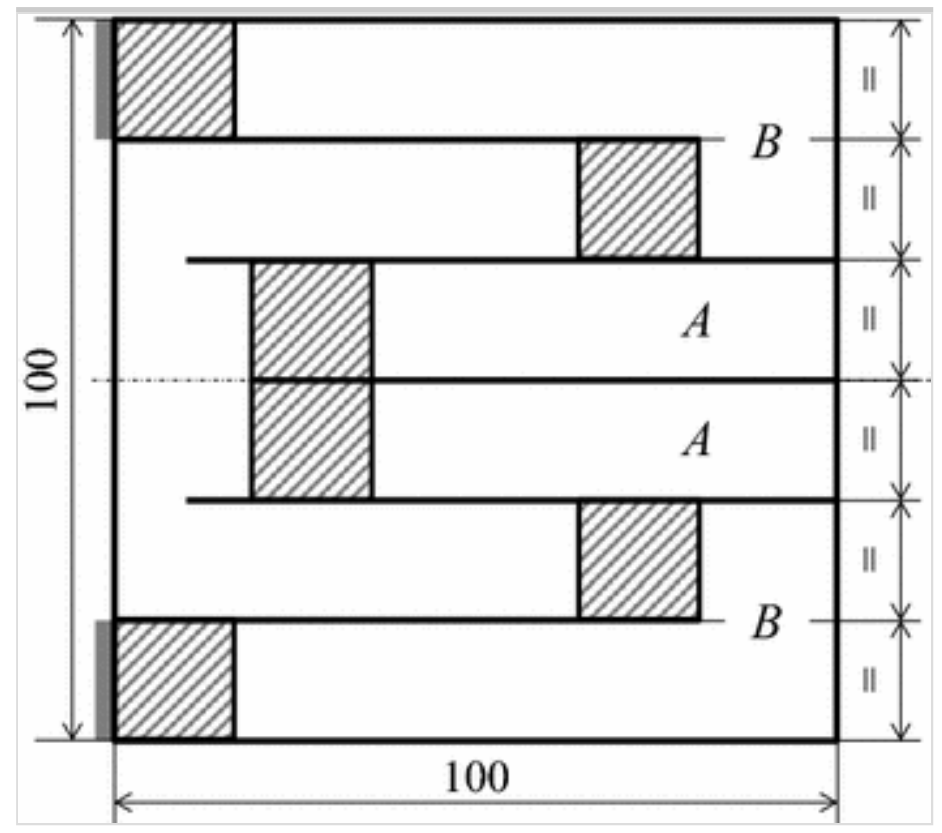

Figure 8 shows the energy harvester prototype made according to the sketch in Fig. 7. In order to simplify the experimental tests, only three piezoelectric transducers were applied on the top left-hand side of the support lamina, in the positions highlighted in Fig. 7. A complete prototype would include three additional piezoelectric transducers on the top right hand side (as in Fig. 7) and the corresponding six transducers on the bottom side of the support lamina.

\section{Fig. 8}

Prototype of the energy harvester fixed on the shaker vibrating table

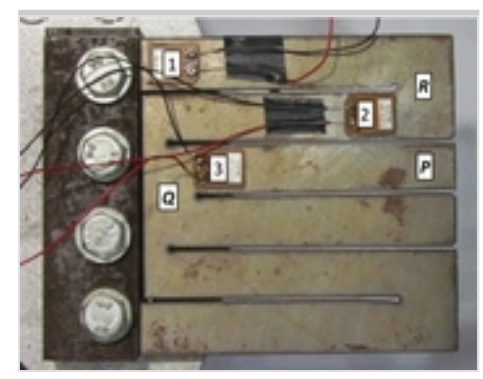


The proposed prototype involves a mild steel support lamina with a side length equal to $100 \mathrm{~mm}$. According to the maximum bending strain areas, three DuraAct piezoelectric transducers P-876.SP1 [31], with dimensions $16 \mathrm{~mm} \times$ $13 \mathrm{~mm} \times 0.5 \mathrm{~mm}$, were used. The piezoelectric transducers were bonded to the support lamina through the Hysol Loctite 3422 A\&B adhesive [32], a bicomponent epoxy adhesive. A couple of cables welded to the solder paths of the transducers ensure the electrical connection.

\subsection{Modal and dynamical analysis of the prototype}

Figure 9 shows the FE model of the converter prototype, which describes in detail the prototype in Fig. 8. This model improves that in the previous Section by including the DuraAct piezoelectric transducers. These transducers are represented by a single layer of piezoelectric hexahedral elements (C3D8E) having the particular feature of an electrical conduction degree of freedom in addition to the translational ones. According to the data provided by the manufacturer [33], an equivalent Young's modulus of 23.3 GPa, and a Poisson's ratio of 0.3 were set for the piezoelectric transducers.

\section{Fig. 9}

FE model of the converter prototype in Fig. 8 with close-up view of the piezoelectric transducer

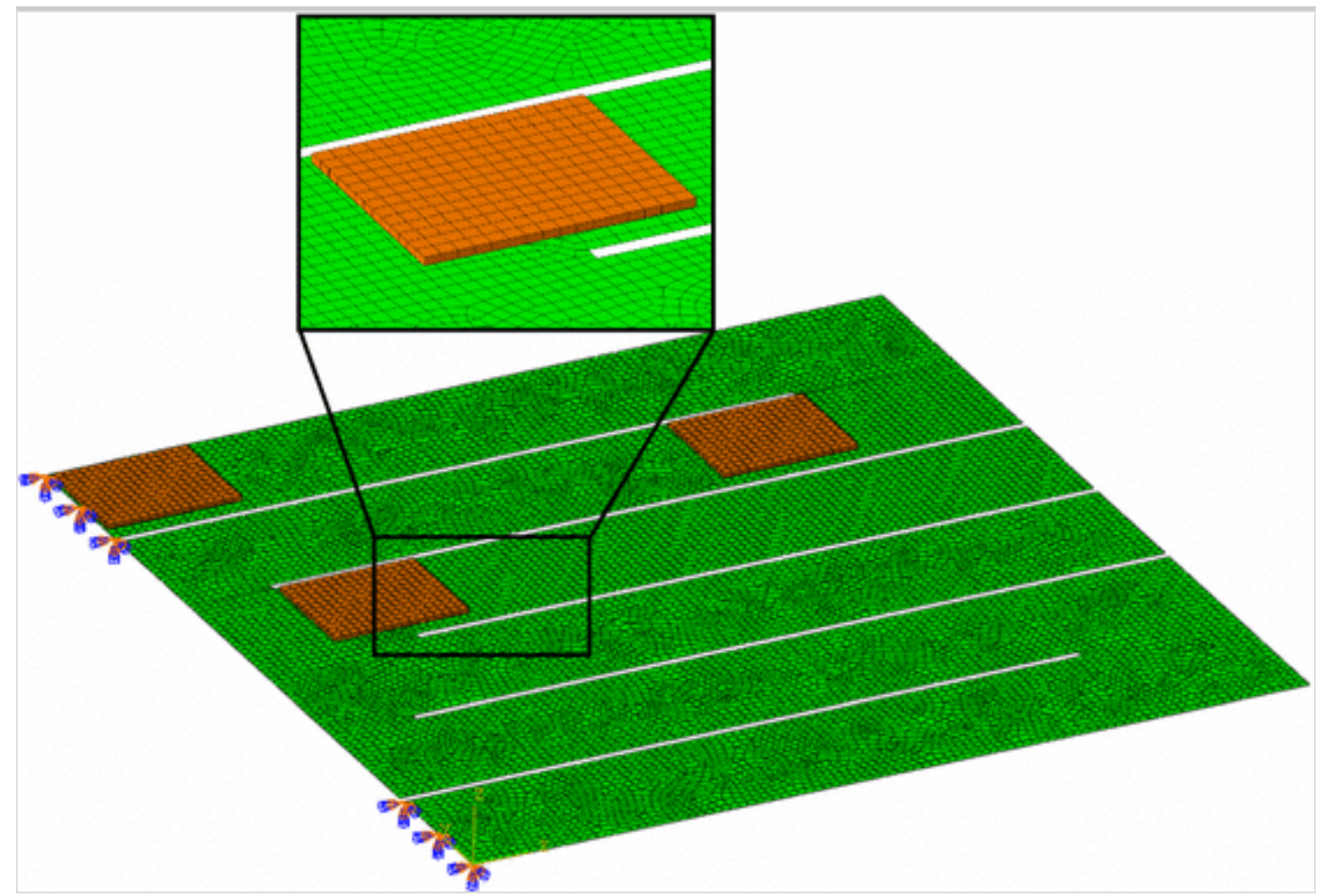


A tied mesh internal kinematic constraint [28], which equals the corresponding degrees of freedom on opposite faces, joins the piezoelectric transducers to the support plate. A built-in boundary condition describes the external constraints on the support plate.

Two are the steps of the analysis. The first step performs a modal analysis in the range between 0 and $100 \mathrm{~Hz}$, through the Lanczos algorithm. In order to investigate the dynamic linearized response of the converter to harmonic excitation, the second step performs a direct-solution steady-state dynamic analysis [28]. This analysis procedure applies to the external constraints an input sinusoidal acceleration equal to $0.3 \mathrm{~g}$ orthogonal to the converter plane, which varies in the frequency range between $0 \mathrm{~Hz}$ and $100 \mathrm{~Hz}$. All the analyses were performed on a notebook equipped with an Intel i7 processor and 16 GB of RAM.

\section{Experimental validation}

The experimental tests on the converter prototype (Fig. 8), aiming to investigate the modal response and the power output respectively, were performed in three steps.

The first step identified the eigenfrequencies of the prototype in the range from $0 \mathrm{~Hz}$ up to $100 \mathrm{~Hz}$, by applying a sinusoidal excitation with a frequency that sweeps from $5 \mathrm{~Hz}$ to $100 \mathrm{~Hz}$, and a peak input acceleration equal to $9.81 \mathrm{~m} / \mathrm{s}^{2}$.

For each of these eigenfrequencies, the second step investigated the peak output voltage and the peak output power from the converter, for the following resistive load values: $6800,1000,470,220,100,47,10 \mathrm{k} \Omega$. In order to limit the tip deflection of the support plate, an input acceleration equal to $2.94 \mathrm{~m} / \mathrm{s}^{2}(0.3 \mathrm{~g})$ was applied.

The third step investigated the effect of a $10 \mathrm{~g}$ mass applied in point $Q$ of the converter prototype (see Fig. 8). The tests were performed as in the previous step, but limited to three resistive loads: 6800,470 , and $100 \mathrm{k} \Omega$.

The experimental set-up for the tests included an electrodynamic shaker (Data Physics BV400 [34]) and a Polytec point laser Doppler vibrometer [35]. The electrodynamic shaker was controlled in closed-loop through a miniature accelerometer (MMF KS94B100 [36]), that was fixed to the vibrating table. An 8-channel Abacus controller [34] together with the Signal Star [34] software allowed both to manage the shaker and to perform data acquisition. The Polytec point laser Doppler vibrometer was equipped with an OFV-505 sensor head and 
controlled by a Polytec OFV-5000 controller [35]. In order to identify the eigenfrequencies of the converter prototype and the corresponding tip deflection, the laser vibrometer was set up vertically, at a distance of $1 \mathrm{~m}$ from the plane of the converter. The speed measurement required a sensitivity equal to $500 \mathrm{~mm} /(\mathrm{s} \mathrm{V})$, and was performed near the tip of cantilever $A$, and $B$ in Fig. 7, in order to exactly identify the eigenfrequencies of the structure. The tip deflection was measured at point $A$, but using a sensitivity equal to $5 \mathrm{~mm} / \mathrm{V}$. The PC, equipped with the Signal Star software, also registered the data from the laser Doppler vibrometer. All the DuraAct piezoelectric transducers on the converter prototype were electrically connected to a 16-channels data acquisition module (USB 6251 from National Instruments [37]). The Labview SignalExpress software, installed on a notebook, managed the data acquisition module and recorded the output voltage from each transducer.

\section{Results}

Table 2 compares the eigenfrequencies predicted by the detailed FE model of the converter prototype (Fig. 9) to those measured experimentally both for the energy converter by itself and with the added mass. For the sake of conciseness, we do not present the eigenmodes of the converter prototype, being nearly identical to those in Fig. 6 observed for the support structure alone.

\section{Table 2}

Eigenfrequencies from the analytical model, FE model (in Fig. 9) and registered experimentally of the converter prototype in the range below $100 \mathrm{~Hz}$, both by itself and with a $10 \mathrm{~g}$ added mass

\begin{tabular}{|c|c|c|c|c|}
\hline \multirow[b]{2}{*}{ Eigenfrequencies } & \multirow{2}{*}{$\begin{array}{l}\text { Analytical model } \\
(\mathrm{Hz})\end{array}$} & \multirow{2}{*}{$\begin{array}{l}\text { FE model } \\
(\mathrm{Hz})\end{array}$} & \multicolumn{2}{|c|}{ Experimental tests $(\mathrm{Hz})$} \\
\hline & & & $\begin{array}{l}\text { No added } \\
\text { mass }\end{array}$ & $\begin{array}{l}\text { Added } \\
\text { mass }\end{array}$ \\
\hline$f_{1}$ & 32.47 & 34.0 & 29.8 & 31.2 \\
\hline$f_{2}$ & & 42.5 & 45.2 & \\
\hline$f_{3}$ & 59.38 & 60.3 & 53.8 & \\
\hline$f_{4}$ & 65.68 & 69.2 & 62.6 & 65.3 \\
\hline$f_{5}$ & & 88.1 & 82.9 & 85.3 \\
\hline
\end{tabular}

Figure 10 displays the experimental tip speed of the converter prototype measured at points $P, Q$, and $R$ (see Fig. 8) in the frequency range below $100 \mathrm{~Hz}$, with an input acceleration equal to $9.81 \mathrm{~m} / \mathrm{s}^{2}$. 
Fig. 10

Experimental tip speed of the converter prototype ( $P, Q$, and $R$ refers to Fig. 8), for an input acceleration equal to $9.81 \mathrm{~m} / \mathrm{s}^{2}$

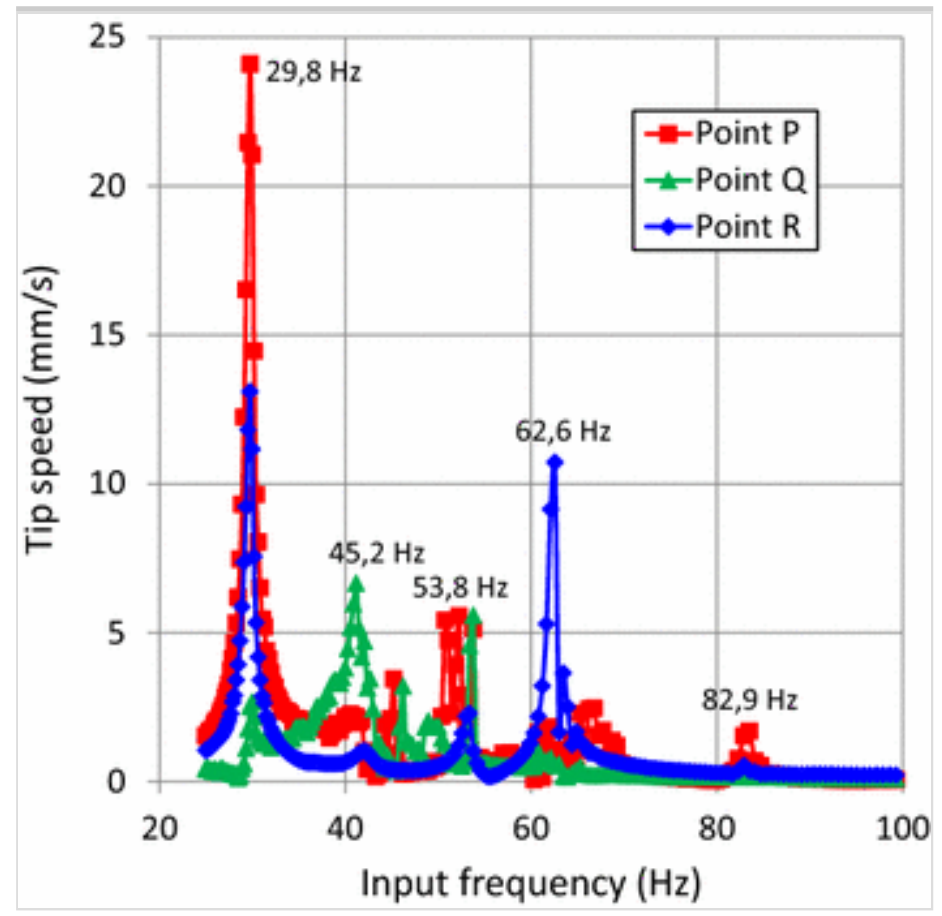

For the same input acceleration and a $6800 \mathrm{k} \Omega$ resistive load applied to the transducers, Fig. 11 compares the tip deflection of the converter prototype provided by the FE model in Fig. 9 to that registered experimentally, either at point $P$ or at point $Q$ (see Fig. 8), for each eigenfrequency.

\section{Fig. 11}

FE prediction (from the model in Fig. 9) and experimental measurements of the tip deflection of the converter prototype ( $P$ and $Q$ refers to Fig. 8), for each eigenfrequency below $100 \mathrm{~Hz}$ and a resistive load equal to $6800 \mathrm{k} \Omega$ 


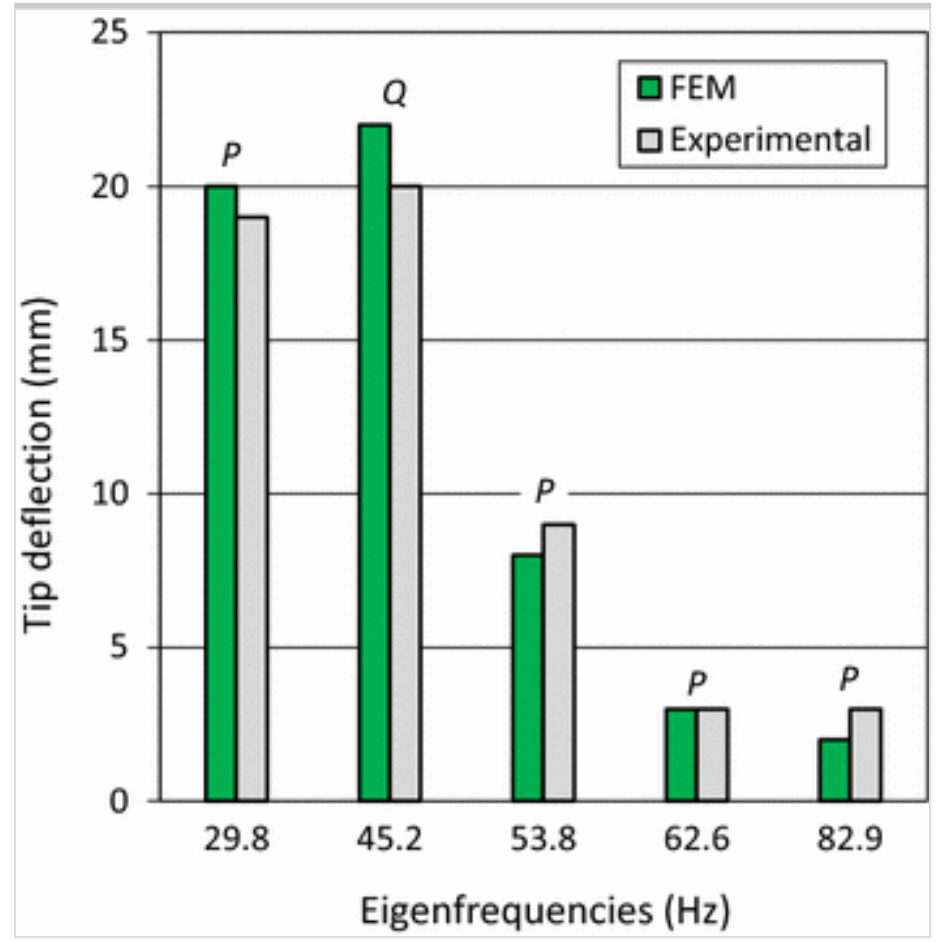

Figure 12 shows the experimental output voltage from the DuraAct piezoelectric transducers as a function of time: the input excitation corresponds to the fundamental frequency of the converter, the input acceleration equals $0.3 \mathrm{~g}$, and a $1000 \mathrm{k} \Omega$ resistive load was applied to each transducer. Each curve refers to one of the transducers, as numbered in Fig. 8.

\section{Fig. 12}

Experimental output voltage versus time of the converter prototype for an input excitation tuned to the fundamental frequency, and with a $1000 \mathrm{k} \Omega$ resistive load

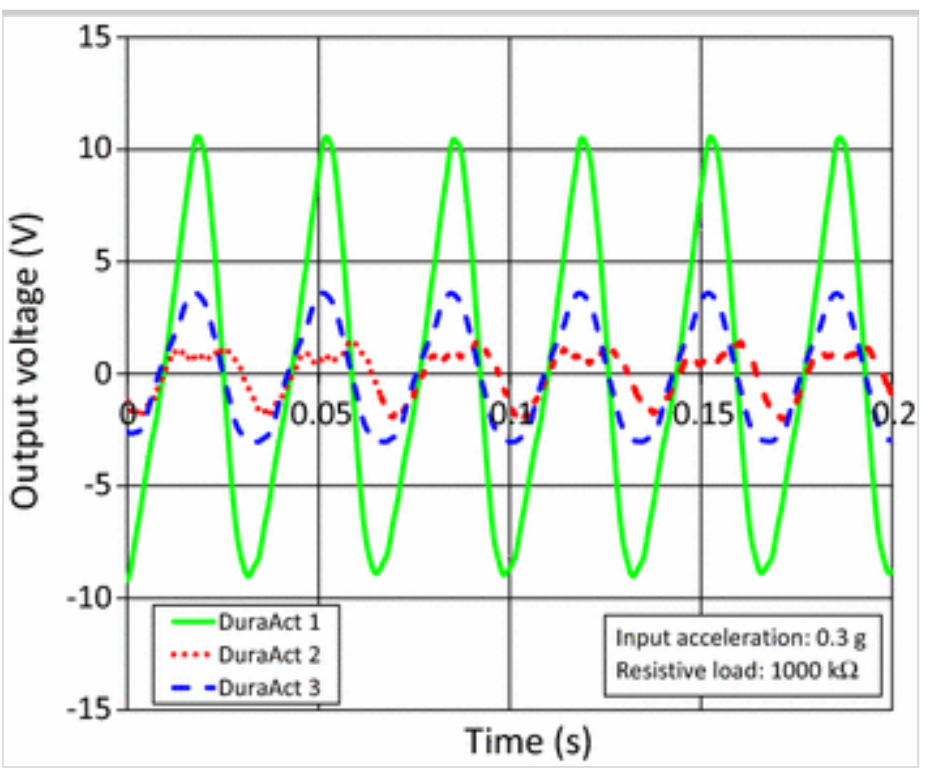


Using the same numbering for the DuraAct transducers, Fig. 13 displays the output root mean square (RMS) voltage predicted by the FE model in Fig. 9 and registered experimentally for each eigenfrequency at different resistive loads applied to each transducer.

\section{Fig. 13}

Output root mean square voltage, $V_{R M S}$, predicted by the FE model (in Fig. 9) and measured experimentally on the converter prototype, for each eigenfrequency and resistive load: DuraAct transducer \#1 (a), \#2 (b), and \#3 (c) 

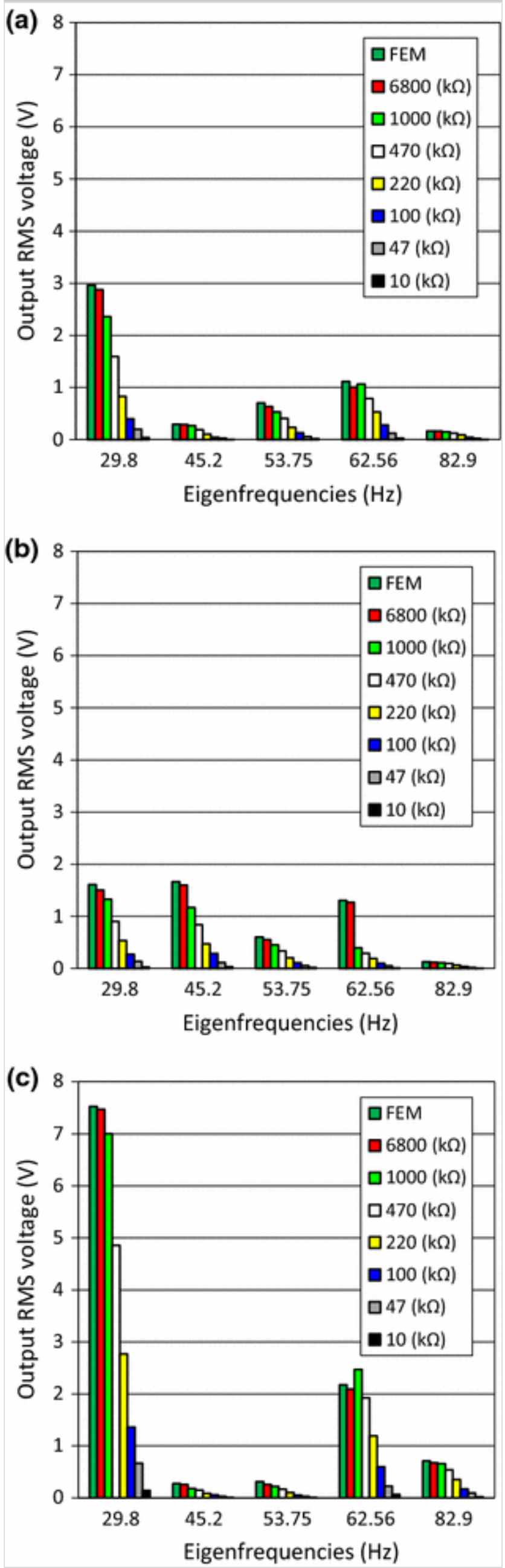
Similarly, Fig. 14 shows the bar charts of the experimental output power from each DuraAct transducer as a function of the eigenfrequency and of the resistive load (from $1000 \mathrm{k} \Omega$ down to $10 \mathrm{k} \Omega$ ). With the same layout, Fig. 15 reports the experimental output power from each transducer of the converter prototype with an added mass in point $Q$ (Fig. 8).

Fig. 14

Output power measured experimentally on the converter prototype for each eigenfrequency and resistive load: DuraAct transducer \#1 (a), \#2 (b), and \#3 (c) 

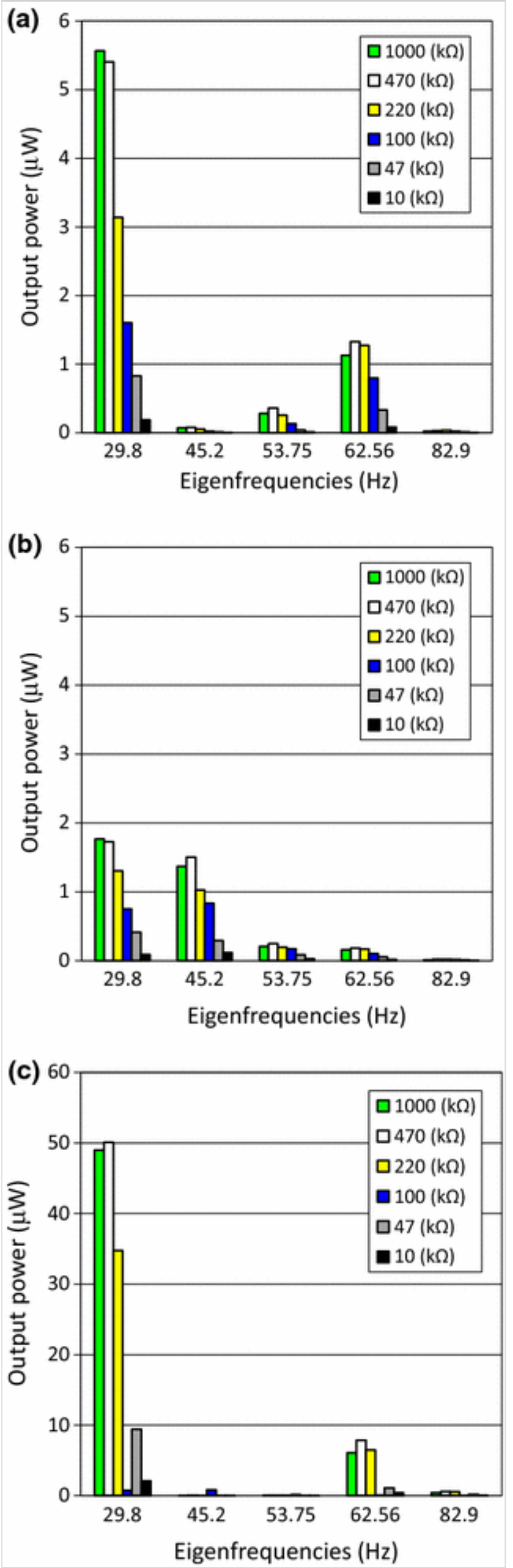
Fig. 15

Output power measured experimentally on the converter prototype with an added mass for each eigenfrequency and resistive load: DuraAct transducer \#1 (a), \#2 (b), and \#3 (c) 

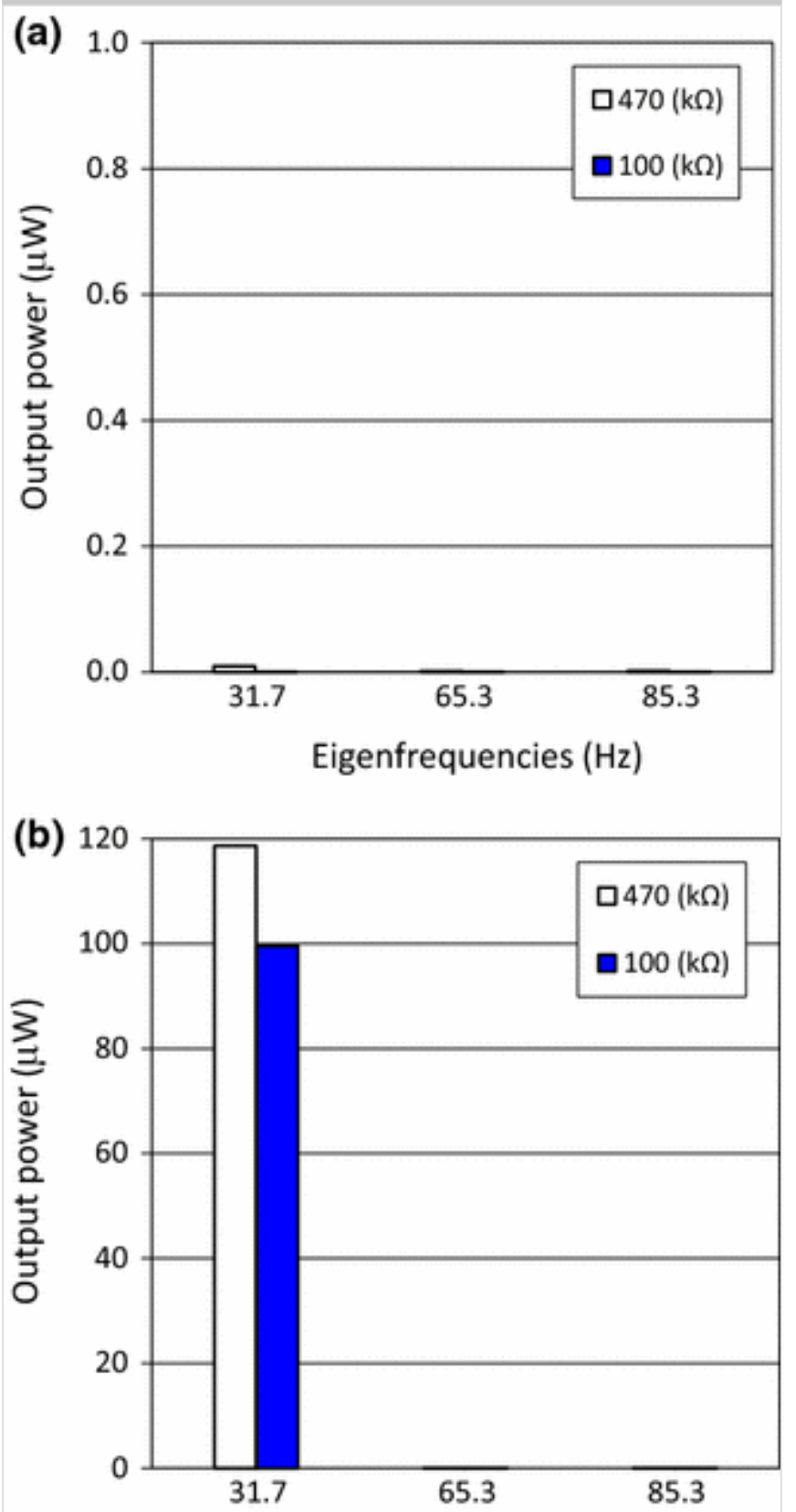

Eigenfrequencies $(\mathrm{Hz})$

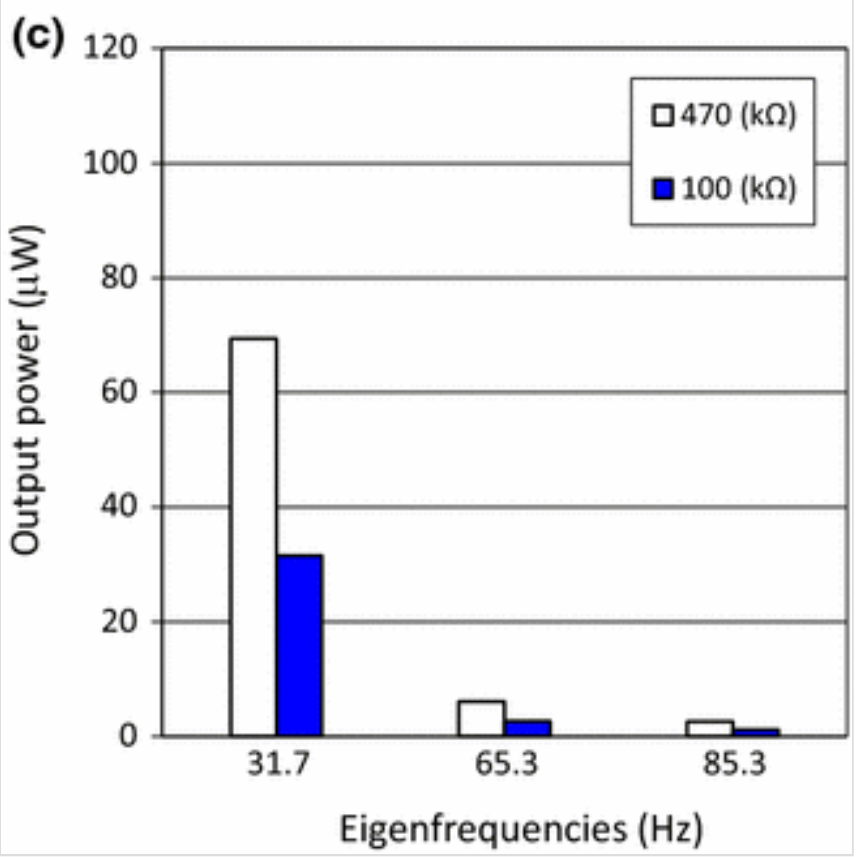


The bar charts in Fig. 16 compare the total output power from the converter prototype without an added mass (Fig. 16a) to that from the converter with an added mass (Fig. 16b). Each bar of the chart sums up the output power from each of the three transducers.

Fig. 16

Total output power (sum of all DuraAct transducers) measured experimentally for each eigenfrequency and resistive load: converter prototype by itself (a), and with an added mass in point $Q(\mathbf{b})$
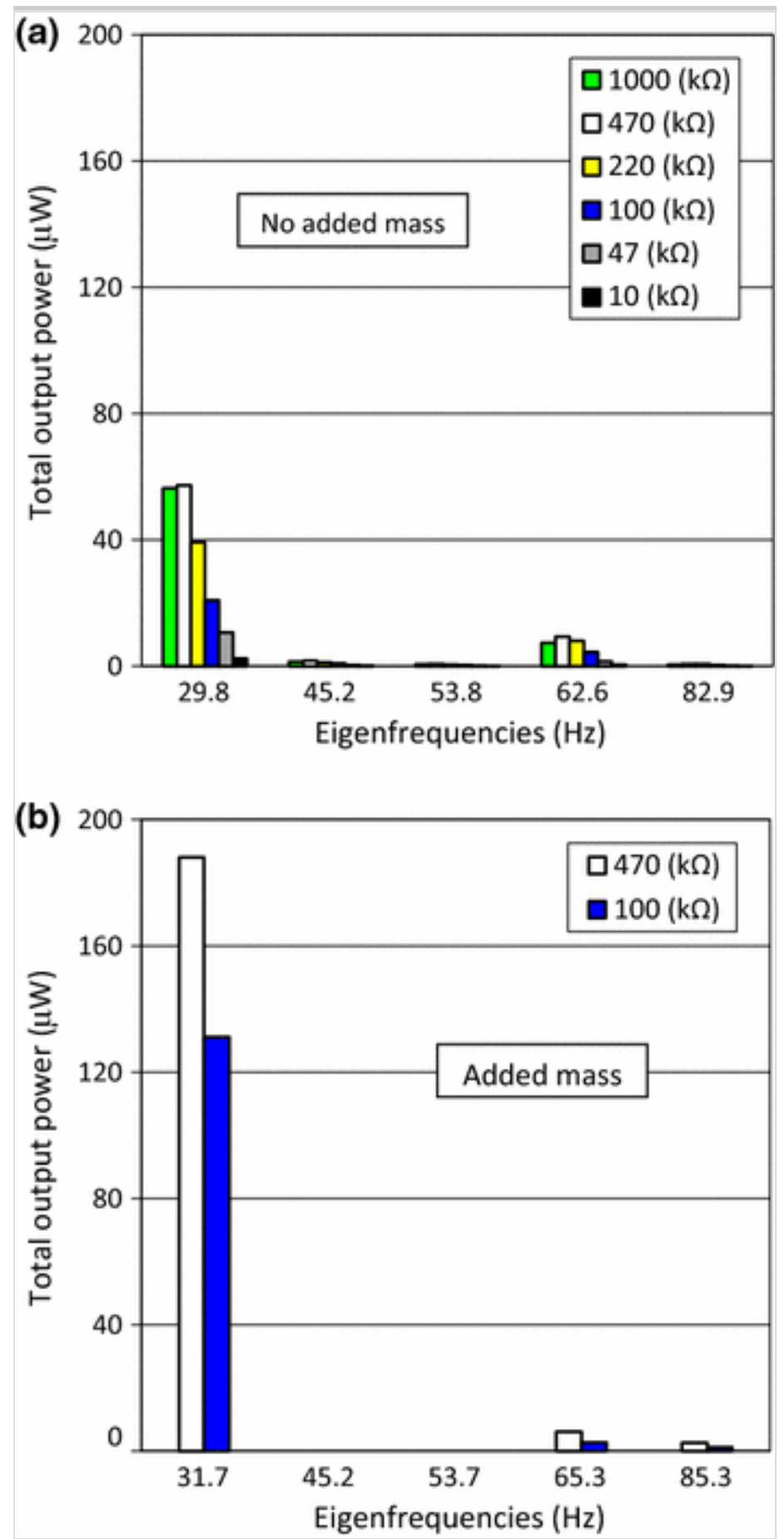


\section{Discussion}

By comparing the prediction from the analytical model, from FE model and the experimental measurements (Table 2), for the converter without an added mass, we can make two observations. First, despite the analytical model neglects the piezoelectric patches, it gives an accurate prediction of the eigenfrequencies registered experimentally on the structure, with exception of the two torsional mode shapes (at 42.5 and $88.1 \mathrm{~Hz}$ ). The remaining three eigenfrequencies are close to the experimental values. Such a feedback allows us to neglect the contribution from the DuraAct piezo patches [33], due to their very low stiffness, in order to provide a simpler but reliable analytical model that may represent a useful design tool for this kind of devices. Second, the FE model predicts the same number of eigenfrequencies registered experimentally below $100 \mathrm{~Hz}$ (Table 2): with exception of the second one, the FE prediction overestimates the experimental eigenfrequency values. This discrepancy may be mainly imputed to the simplified model of the DuraAct transducers, which were described in terms of homogeneous equivalent elastic properties, both with regard to the thickness and to the longitudinal direction. The introduction of an added mass where the inner cantilevers are constrained on the dynamic magnifier structure affects only the second and third eigenfrequencies registered below $100 \mathrm{~Hz}$ that become equal to the fourth and fifth eigenfrequencies of the original converter, respectively.

The experimental tip speed curves in Fig. 10 clearly show that the bending and twisting deformations induced by each eigenmode attain the maximum values at different regions of the structure. The fundamental eigenmode is the easiest to identify, since it clearly involves each of the three measurement points.

The bar chart in Fig. 11 displays that the highest values of the tip deflection occur for the first and second eigenmodes at points $P$ and $Q$, respectively (Fig. 8). For the higher eigenmodes, the tip deflection remarkably decreases, and the most significant values are always registered at point $P$.

The curves in Fig. 12 highlight that, despite the low input acceleration, the output voltage generation from the piezoelectric transducers is remarkable and in phase, in particular from the number \#1 (up to $10 \mathrm{~V}$ ) and \#3 (up to $4 \mathrm{~V}$ ). By contrast, the ripples in the dotted curve (number \#2) is probably imputable to a very low strain occurring at this piezoelectric patch for this eigenmode.

Three observations can be made from Fig. 13. First, for each eigenfrequency the output RMS voltage is significant, either for all the three DuraAct transducers (see in particular the fundamental eigenfrequency and the fourth eigenfrequency), or for some of them. Second, the FE prediction from the model 
in Fig. 9, corresponding to an open circuit model, closely agrees with the experimental values measured for a $6800 \mathrm{k} \Omega$ resistive load. Third, for all the eigenfrequencies, the output RMS voltage remarkably decreases as the resistive load decreases.

The bar charts of the output power in Fig. 14 highlight that the fundamental eigenfrequency exhibits the highest output power value, since all the three transducers are simultaneously active. By comparing the transducers, it appears that the number \#3 (Fig. 14c), which is applied to the inner cantilever (Fig. 8), provides the highest output power values, in particular at the first and fourth eigenfrequencies. By contrast, the output power from transducers \#1 and \#2 (Fig. 14a, b), which are applied to the dynamic magnifier part of the structure (Fig. 8), is nearly an order of magnitude lower than from number \#3. This is clearly imputable to the lower bending strain occurring on this $S$-shaped part of the structure.

If we focus on the bar charts in Fig. 15, it clearly appears that the added mass on the converter prevents the power generation on the piezoelectric transducer \#1 (see Fig. 8) for all the three eigenfrequencies examined. This occurrence is due to rigid behavior of the outer part of the dynamic magnifier. The highest output power (up to $120 \mu \mathrm{W}$ ) was registered from the piezoelectric transducer \#2 (see Fig. 8) at the fundamental frequency, but for subsequent eigenfrequencies the output power of this transducer becomes zero. Transducer \#3 exhibited approximately half the output power of \#2 at the fundamental frequency, while providing a significant output power also at subsequent eigenfrequencies.

By examining the total output power provided by the prototype without an added mass (Fig. 16a) it clearly appears that the highest conversion occurs at the first and fourth eigenfrequencies, reaching nearly 60 and $10 \mu \mathrm{W}$ respectively. A remarkably lower but not negligible output power occurs also at the other eigenfrequencies, where a total of some microwatts was registered. In case of the prototype with the added mass (Fig. 16b), the total output power at the fundamental frequency is about three time higher than for the original converter, while at subsequent eigenfrequencies the two converters provide nearly the same values. On the whole, the added mass shifts some eigenfrequencies of the system out of the control window. However, it largely increases the efficiency of the converter in particular at the fundamental frequency. Regardless the added mass, it appears that the $470 \mathrm{k} \Omega$ is the optimal resistive load for all the eigenfrequencies here examined.

As general observation, the proposed energy converter relying on dynamic magnification appears promising since it provides many eigenfrequencies below 
$100 \mathrm{~Hz}$ and a significant output power. If needed, it is possible to tune the converter to the desired frequency range by acting on the length and thickness of the support lamina (as described in Table 1), or to improve the efficiency at some specific eigenfrequencies by introducing a small added mass. In order to increase the output power, it is possible to apply three additional piezoelectric transducers on the symmetric half of the top surface, as outlined in Fig. 7, but also to mirror the same configuration of the transducers on the bottom surface.

The proposed solution compares well to similar solutions from the literature [7, $23,25,26,27,38]$ both with regard to the eigenfrequencies and to the output power.

\section{Conclusions}

The paper presented a simple and innovative piezoelectric energy harvester based on a square support lamina. The converter features a couple of dynamic magnifiers that support two cantilevers, for a total side length of the structure equal to $100 \mathrm{~mm}$. The paper proposed a simple analytical model to investigate the eigenfrequencies of the proposed structure. In addition, a detailed computational analysis was developed to take into account the effect of the piezoelectric transducers. The experimental validation confirmed a quite valuable frequency response including five eigenfrequencies below $100 \mathrm{~Hz}$. The total output power from the three piezoelectric transducers here used was significant, in particular over two of the five eigenfrequencies of the system. The application of a small ballast mass showed that the converter can be tuned at some of its eigenfrequencies, improving its conversion efficiency. In addition, the eigenfrequencies range can be significantly shifted up or down either by choosing an appropriate thickness for the support lamina or a desired side length.

Compliance with ethical standards

Conflict of interest The authors declare that they have no conflict of interest.

\section{References}

1. Aldraihem O, Baz A (2011) Energy harvester with a dynamic magnifier. J Intell Mater Syst Struct 22(6):521-530

2. Cornwell PJ (2005) Enhancing power harvesting using a tuned auxiliary structure. J Intell Mater Syst Struct 16(10):825-834 
3. Rastegar J, Haarhoff D, Pereira C, Nguyen H-L (2006) Piezoelectric-based energy harvesting power sources for gun-fired munitions. Smart Struct Mater 6174:61740W-61740W-6

4. Ma PS, Kim JE, Kim YY (2010) Power-amplifying strategy in vibrationpowered energy harvesters. In: Proceedings of SPIE 7643, active and passive smart structures and integrated systems

5. Lee S, Youn BD, Jung BC (2009) Robust segment-type energy harvester and its application to a wireless sensor. Smart Mater Struct 18(9):95021

6. Yang Z, Yang J (2009) Connected vibrating piezoelectric bimorph beams as a wide-band piezoelectric power harvester. J Intell Mater Syst Struct 20(5):569-574

7. Erturk A, Renno JM, Inman D (2009) Modeling of piezoelectric energy harvesting from an L-shaped beam-mass structure with an application to UAVs. J Intell Mater Syst Struct 20:529-544

8. Xu JW, Shao WW, Kong FR, Feng ZH (2010) Right-angle piezoelectric cantilever with improved energy harvesting efficiency. Appl Phys Lett 96(15):152904

9. Tang X, Zuo L (2011) Enhanced vibration energy harvesting using dualmass systems. J Sound Vib 330(21):5199-5209

10. Aladwani A, Arafa M, Aldraihem O, Baz A (2012) Cantilevered piezoelectric energy harvester with a dynamic magnifier. J Vib Acoust 134(3):31004

11. Aladwani A, Aldraihem O, Baz A (2014) A distributed parameter cantilevered piezoelectric energy harvester with a dynamic magnifier. Mech Adv Mater Struct 21:566-578

12. Nouh M, Aldraihem O, Baz A (2012) Energy harvesting of thermoacoustic-piezo systems with a dynamic magnifier. J Vib Acoust 134(6):61015

13. Seo M-H, Choi D-H, Kim I-H, Jung H-J, Yoon J-B (2012) Multi-resonant energy harvester exploiting high-mode resonances frequency down-shifted by a flexible body beam. Appl Phys Lett 101:123903-1-4 
14. Zhou W, Penamalli GR, Zuo L (2012) An efficient vibration energy harvester with a multi-mode dynamic magnifier. Smart Mater Struct 21:15014

15. Vasic D, Costa F (2013) Modelling of piezoelectric energy harvester with multi-mode dynamic magnifier with matrix representation. Int J Appl Electromagn Mech 43:237-255

16. Dhakar L, Liu H, Tay FEH, Lee C (2013) A new energy harvester design for high power output at low frequencies. Sens Actuators A Phys 199:344352

17. Kim JE, Kim YY (2013) Power enhancing by reversing mode sequence in tuned mass-spring unit attached vibration energy harvester. AIP Adv 3(7):72103

18. Sun KH, Kim Y-C, Kim JE (2014) A small-form-factor piezoelectric vibration energy harvester using a resonant frequency-down conversion. AIP Adv 4(10):107125

19. Sharma VK, Srikanth K, Viswanath AK (2014) Influence of crosssectional area of a dynamic magnifier for vibration energy harvesting. In: Proceedings of 4th IRF international conference, vol 1, pp 69-72

20. O’Donoghue D, Nico V, Frizzell R, Kelly G, Punch J (2014) A multipledegree-of-freedom velocity-amplified vibrational energy harvester part a: experimental analysis. In: ASME 2014 conference on smart materials, adaptive structures and intelligent systems, SMASIS 2014, vol 2

21. Nico V, O’Donoghue D, Frizzell R, Kelly G, Punch J (2014) A multiple degree-of-freedom velocity-amplified vibrational energy harvester: part $\mathrm{b}$ : modelling. In: ASME 2014 conference on smart materials, adaptive structures and intelligent systems, SMASIS 2014, vol 2

22. Nico V, Boco E, Frizzell R, Punch J (2016) A high figure of merit vibrational energy harvester for low frequency applications. Appl Phys Lett 108(1):013902

23. Castagnetti D (2011) Fractal-inspired multifrequency structures for piezoelectric harvesting of ambient kinetic energy. J Mech Des 133(11):111005 
24. Castagnetti D (2015) A piezoelectric based energy harvester with dynamic magnification. In: ASME 2015 conference on smart materials, adaptive structures and intelligent systems volume 2: integrated system design and implementation; structural health monitoring; bioinspired smart materials and systems; energy harvesting, p V002T07A001

25. Castagnetti D (2012) Experimental modal analysis of fractal-inspired multi-frequency structures for piezoelectric energy converters. Smart Mater Struct 21(9):94009

26. Castagnetti D (2013) A wideband fractal-inspired piezoelectric energy converter: design, simulation and experimental characterization. Smart Mater Struct 22:94024

27. Castagnetti D (2015) Comparison between a wideband fractal-inspired and a traditional multicantilever piezoelectric energy converter. J Vib Acoust 137(1):11006

28. Simulia (2012) SIMULIA ABAQUS, user's manual. Dassault Systèmes Simulia Corp., Providence, RI, USA

29. Kelly SG (2000) Fundamentals of mechanical vibrations, 2nd edn. McGraw-Hill Science, New York

30. Krodkiewski JM (2008) Mechanical vibration. The University of Melbourne, Parkville

31. P. P. Technology (2015) No title. P-876 DuraAct Patch Transducer, 2015. http://piceramic.com/product-detail-page/p-876-101790.html. Accessed: 12 Jan 2015

32. Park TB (2015) Technical data sheet Hysol ${ }^{\circledR} 3422,2003$.

https://www.kaindltech.at/fileadmin/Datenblaetter/Datenblaetter/Loctite/3422EN.pdf. Accessed: 12 Jan 2015

33. Piezo PI, Technology C, S. P. Com-, Piezoelectric patch transducers for industry and research key technologies under one roof : a plus for our customers

34. Electrodynamic Shaker (2018) http://www.dataphysics.com/. Accessed 18 Jan 2018 
35. Polytech AM (2015) Laser doppler vibrometer.

http://www.polytec.com/eu/. Accessed 19 Jan 2015

36. Miniature Accelerometers (2018) www.mmf.de. Accessed 18 Jan 2018

37. Labview software (2018) www.ni.com/labview/. Accessed 19 Jan 2015

38. Roundy S, Wright PK (2004) A piezoelectric vibration based generator for wireless electronics. Smart Mater Struct 13(5):1131-1142 